\title{
Basics of Lanthanide Photophysics
}

\author{
Jean-Claude G. Bünzli and Svetlana V. Eliseeva
}

\begin{abstract}
The fascination for lanthanide optical spectroscopy dates back to the $1880 \mathrm{~s}$ when renowned scientists such as Sir William Crookes, LeCoq de Boisbaudran, Eugène Demarçay or, later, Georges Urbain were using luminescence as an analytical tool to test the purity of their crystallizations and to identify potential new elements. The richness and complexity of lanthanide optical spectra are reflected in an article published in 1937 by J.H. van Vleck: The Puzzle of Rare Earth Spectra in Solids. After this analytical and exploratory period, lanthanide unique optical properties were taken advantage of in optical glasses, filters, and lasers. In the mid-1970s, E. Soini and I. Hemmilä proposed lanthanide luminescent probes for time-resolved immunoassays (Soini and Hemmilä in Clin Chem 25:353-361, 1979) and this has been the starting point of the present numerous bio-applications based on optical properties of lanthanides. In this chapter, we first briefly outline the principles underlying the simplest models used for describing the electronic structure and spectroscopic properties of trivalent lanthanide ions $\mathrm{Ln}^{\mathrm{III}}\left(4 \mathrm{f}^{n}\right)$ with special emphasis on luminescence. Since the book is intended for a broad readership within the sciences, we start from scratch defining all quantities used, but we stay at a descriptive level, leaving out detailed mathematical developments. For the latter, the reader is referred to references Liu and Jacquier, Spectroscopic properties of rare earths in optical materials. Tsinghua University Press \& Springer, Beijing \& Heidelberg, 2005 and Görller-Walrand and Binnemans, Rationalization of crystal field parameters. In: Gschneidner, Eyring (eds) Handbook on the physics and chemistry of rare earths, vol 23. Elsevier BV, Amsterdam, Ch 155, 1996. The second part of the chapter is devoted to practical aspects of lanthanide luminescent probes, both from the point of view of their design and of their potential utility.
\end{abstract}

J.-C.G. Bünzli $(\bowtie)$ and S.V. Eliseeva

Laboratory of Lanthanide Supramolecular Chemistry, École Polytechnique Fédérale de Lausanne, BCH 1402, 1015 Lausanne, Switzerland

e-mail: Jean-Claude.Bunzli@epfl.ch 
Keywords Crystal-field analysis · Energy transfer · f-f Transition · Intrinsic quantum yield . Lanthanide bioprobe - Lanthanide luminescence · Lanthanide spectroscopy · Lifetime - Luminescence sensitization - Population analysis · Quantum yield · Radiative lifetime $\cdot$ Selection rule $\cdot$ Site symmetry $\cdot$ Stern-Volmer quenching

\section{Contents}

1 Electronic Structure of Trivalent Lanthanide Ions

1.1 Atomic Orbitals

1.2 Electronic Configuration

1.3 The Ions in a Ligand Field

2 Absorption Spectra

2.1 Induced ED f-f Transitions: Judd-Ofelt Theory [5, 6]

$2.24 \mathrm{f}-5 \mathrm{~d}$ and CT Transitions

3 Emission Spectra

4 Sensitization of Lanthanide Luminescence

4.1 Design of Efficient Lanthanide Luminescent Bioprobes

4.2 Practical Measurements of Absolute Quantum Yields

5 Information Extracted from Lanthanide Luminescent Probes

5.1 Metal Ion Sites: Number, Composition, and Population Analysis

5.2 Site Symmetry Through Crystal-Field Analysis

5.3 Strength of Metal-Ligand Bonds: Vibronic Satellite Analysis

5.4 Solvation State of the Metal Ion

5.5 Energy Transfers: Donor-Acceptor Distances and Control of the Photophysical Properties of the Acceptor by the Donor

5.6 FRET Analysis

5.7 Ligand Exchange Kinetics

5.8 Analytical Probes

6 Appendices

6.1 Site Symmetry Determination from Eu ${ }^{\mathrm{III}}$ Luminescence Spectra

6.2 Examples of Judd-Ofelt Parameters

6.3 Examples of Reduced Matrix Elements

6.4 Emission Spectra

References

\section{Abbreviations}

$\begin{array}{ll}\text { AO } & \text { Acridine orange } \\ \text { CF } & \text { Crystal field } \\ \text { CT } & \text { Charge transfer } \\ \text { DMF } & \text { Dimethylformamide } \\ \text { DNA } & \text { Deoxyribonucleic acid } \\ \text { dpa } & \text { Dipicolinate (2,6-pyridine dicarboxylate) } \\ \text { dtpa } & \text { Diethylenetrinitrilopentaacetate } \\ \text { EB } & \text { Ethidium bromide } \\ \text { ED } & \text { Electric dipole }\end{array}$




$\begin{array}{ll}\text { EQ } & \text { Electric quadrupole } \\ \text { FRET } & \text { Förster resonant energy transfer } \\ \text { hfa } & \text { Hexafluoroacetylacetonate } \\ \text { ILCT } & \text { Intraligand charge transfer } \\ \text { ISC } & \text { Intersystem crossing } \\ \text { JO } & \text { Judd-Ofelt } \\ \text { LLB } & \text { Lanthanide luminescent bioprobe } \\ \text { LMCT } & \text { Ligand-to-metal charge transfer } \\ \text { MD } & \text { Magnetic dipole } \\ \text { MLCT } & \text { Metal-to-ligand charge transfer } \\ \text { NIR } & \text { Near-infrared } \\ \text { PCR } & \text { Polymerase chain reaction } \\ \text { SO } & \text { Spin-orbit } \\ \text { tta } & \text { Thenoyltrifluoroacetylacetonate } \\ \text { YAG } & \text { Yttrium aluminum garnet }\end{array}$

\section{Electronic Structure of Trivalent Lanthanide Ions}

\subsection{Atomic Orbitals}

In quantum mechanics, three variables depict the movement of the electrons around the positively-charged nucleus, these electrons being considered as waves with wavelength $\lambda=h / m v$ where $h$ is Planck's constant $\left(6.626 \times 10^{-34} \mathrm{~J} \mathrm{~s}^{-1}\right), m$ and $v$ the mass $\left(9.109 \times 10^{-31} \mathrm{~kg}\right)$ and velocity of the electron, respectively:

- The time-dependent Hamiltonian operator $\mathcal{H}$ describing the sum of kinetic and potential energies in the system; it is a function of the coordinates of the electrons and nucleus.

- The wavefunction, $\Psi_{n}$, also depending on the coordinates and time, related to the movement of the particles, and not directly observable; its square $\left(\Psi_{n}\right)^{2}$ though gives the probability that the particle it describes will be found at the position given by the coordinates; the set of all probabilities for a given electronic $\Psi_{n}$, is called an orbital.

- The quantified energy $E_{\mathrm{n}}$ associated with a specific wavefunction, and independent of the coordinates.

These quantities are related by the dramatically simple Schrödinger equation, which replaces the fundamental equations of classical mechanics for atomic systems:

$$
\mathcal{H} \Psi_{n}=\mathrm{E}_{\mathrm{n}} \Psi_{n}
$$

Energies $E_{\mathrm{n}}$ are eigenvalues of $\Psi_{n}$, themselves called eigenfunctions. In view of the complexity brought by the multidimensional aspect of this equation 
( 3 coordinates for each electron and nucleus, in addition to time) several simplifications are made. Firstly, the energy is assumed to be constant with time, which removes one coordinate. Secondly, nuclei being much heavier than electrons, they are considered as being fixed (Born-Oppenheimer approximation). Thirdly, since the equation can only be solved precisely for the hydrogen atom, the resulting hydrogenoid or one-electron wavefunction is used for the other elements, with a scaling taking into account the apparent nucleus charge, i.e., including screening effects from the other electrons. Finally, to ease solving the equation for non- $\mathrm{H}$ atoms, the various interactions occurring in the electron-nucleus system are treated separately, in order of decreasing importance (perturbation method).

For hydrogen, the Hamiltonian simply reflects Coulomb's attraction between the nucleus and the electron, separated by a distance $r_{i}$, and the kinetic energy of the latter: ${ }^{1}$

$$
\mathcal{H}_{0}=-\frac{1}{r_{i}}-\frac{1}{2} \Delta_{i} \quad\left(\Delta=\frac{\partial^{2}}{\partial x^{2}}+\frac{\partial^{2}}{\partial y^{2}}+\frac{\partial^{2}}{\partial z^{2}}\right) .
$$

Each wavefunction (or orbital: the two terms are very often, but wrongly, taken as synonyms) resulting from solving (1) is defined by four quantum numbers reflecting the quantified energy of the two motions of the electrons: the orbital motion, defined by the angular momentum $\vec{\ell}$, and the spin, characterized by the angular momentum $\vec{s}$. If polar coordinates $(r, \vartheta, \varphi)$ are used, wavefunctions are expressed as the product of a normalizing factor $N$, of a radial function $\Re_{n, \ell}$, of an angular function $\Phi_{\ell, m_{\ell}}$, and of a spin function $S_{m_{s}}$ :

$$
\Psi_{n, \ell, m_{\ell}, m_{s}}=N \cdot \Re_{n, \ell}(r) \cdot \Phi_{\ell, m_{\ell}}(\vartheta, \varphi) \cdot S_{m_{s}} .
$$

The principal quantum number $n$ is an integer $(1,2,3, \ldots)$ and represents the radial expansion of the orbital. The angular quantum number $\ell$ varies from 0 to $(n-1)$ and characterizes the shape of the orbital (designed by letters: $s, p, d, f, g, \ldots$ for $\ell=0,1,2,3,4, \ldots)$. The magnetic quantum number $m_{\ell}$ is the projection of the vector $\vec{\ell}$ onto the $z$ axis and is linked to the orientation of the orbital in space; it varies between $-\ell$ and $+\ell$. Finally, $m_{s}$ is the projection of the vector $\vec{s}$ and takes values of $\pm 1 / 2$. Pauli's principle requires that two electrons of the same atom must at least differ by the value of one quantum number; this implies that only two electrons of opposite spin can be associated with a given orbital. An electronic shell consists in all electrons having the same quantum number $n$. A sub-shell regroups electrons with same $n$ and $\ell$ numbers, has therefore $(2 \ell+1)$ orbitals, and may contain a maximum of $(4 \ell+2)$ electrons. The shapes of the seven $4 \mathrm{f}$ orbitals $(n=4, \ell=3)$ are represented on top of Fig. 1.

\footnotetext{
${ }^{1}$ We use the atomic system units (a.u.) in order to simplify the equations as much as possible.
} 

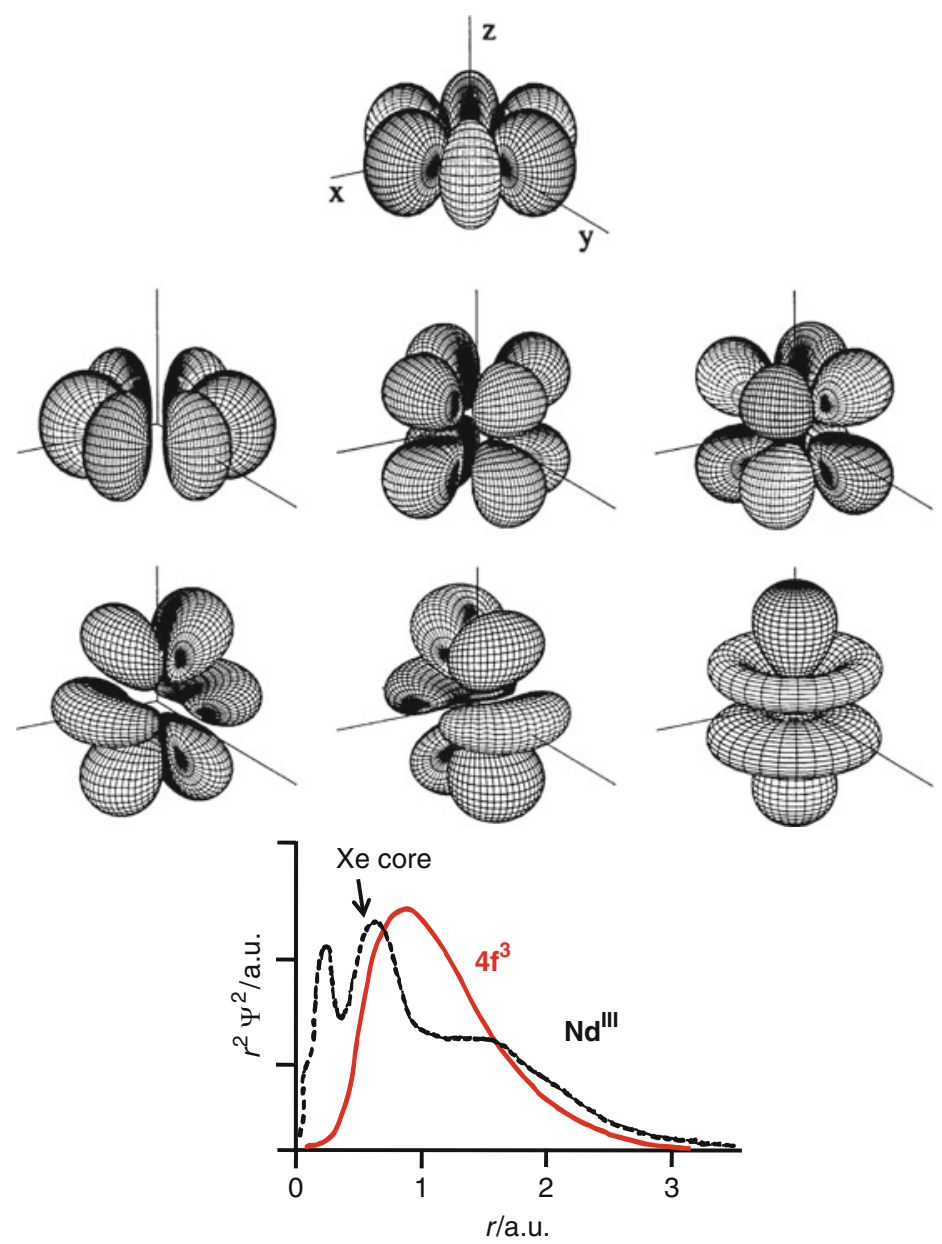

Fig. 1 Top: Shape of the one-electron (hydrogenoid) 4f orbitals in a Cartesian space. From top to bottom and left to right: $4 \mathrm{f}_{\mathrm{x}\left(\mathrm{x}^{2}-3 \mathrm{y}^{2}\right)}, 4 \mathrm{f}_{\mathrm{y}\left(3 \mathrm{y}^{2}-\mathrm{x}^{2}\right)}, 4 \mathrm{f}_{\mathrm{xyz}}, 4 \mathrm{f}_{\left.\mathrm{z}^{2} \mathrm{x}^{2}-\mathrm{y}^{2}\right)}, 4 \mathrm{f}_{\mathrm{xz}^{2}}, 4 \mathrm{f}_{\mathrm{yz}^{2}}$, and $4 \mathrm{f}_{\mathrm{z}^{3}}($ combinations of Cartesian coordinates represent the angular functions). Bottom: Radial wavefunction of the three $4 \mathrm{f}$ electrons of $\mathrm{Nd}^{\mathrm{III}}$ compared with the radial wavefunction of the xenon core (a.u. $=$ atomic units); redrawn after [1]

\subsection{Electronic Configuration}

The ground state electronic configuration of $\mathrm{Ln}^{\mathrm{III}}$ ions is $[\mathrm{Xe}] 4 \mathrm{f}^{n}(n=0-14)$. It is energetically well separated from the $[\mathrm{Xe}] 4 \mathrm{f}^{n-1} 5 \mathrm{~d}^{1}$ configuration $(\Delta E$ $>32,000 \mathrm{~cm}^{-1}$ ). A far reaching fact is the shielding of the $4 \mathrm{f}$ orbitals by the xenon core (54 electrons), particularly the larger radial expansion of the $5 \mathrm{~s}^{2} 5 \mathrm{p}^{6}$ subshells, making the valence $4 \mathrm{f}$ orbitals "inner orbitals" (bottom of Fig. 1). This is the key to the chemical and spectroscopic properties of these metal ions. 
Each of the $n$ electrons of the $4 \mathrm{f}^{n}$ configuration is associated with one of the seven $4 \mathrm{f}$ wavefunctions and may have a spin of $\pm 1 / 2$. There are a number of ways of associating the $n$ electrons with the 4 f orbitals, taking the spin into consideration; this number corresponds to the multiplicity (or degeneracy) of the configuration and is given by the following combinatorial formula:

$$
\frac{(4 \ell+2) !}{n !(4 \ell+2-n) !}=\frac{14 !}{n !(14-n) !} \quad \text { if } \ell=3 \text {. }
$$

Since there are more than one electron in the configuration, the Hamiltonian in (2) has to be adjusted to take into account the number of electrons, the apparent (screened) nucleus charge $Z^{\prime}$, and the repulsion between electrons located at a distance $r_{i j}$ :

$$
\mathcal{H}=\sum_{i=1}^{n}\left(-\frac{Z^{\prime}}{r_{i}}-\frac{1}{2} \Delta_{i}\right)+\sum_{i \neq j}^{n} \frac{1}{r_{i j}}
$$

A given association of the electrons with the $4 \mathrm{f}$ wavefunctions $\left\{\left(m_{\ell}, m_{s}\right)_{1},\left(m_{\ell}\right.\right.$, $\left.\left.m_{s}\right)_{2}, \ldots\left(m_{\ell}, m_{S}\right)_{\mathrm{n}}\right\}$ is called a micro state. The latter is characterized with "overall" quantum numbers $M_{L}$ and $M_{S}$ derived from the projections of the sum of the angular momenta:

$$
\vec{L}=\sum_{i=1}^{n} \vec{\ell}_{i}, \quad \vec{S}=\sum_{i=1}^{n} \vec{s}_{i}, \quad M_{L}=\sum_{i=1}^{n}\left(m_{\ell}\right)_{i}, \quad M_{S}=\sum_{i=1}^{n}\left(m_{s}\right)_{i}
$$

A set of micro states such that all the $M_{L}$ and $M_{S}$ quantum numbers correspond to the projections of one value of $L$ and $S$, respectively, is called a spectroscopic term. It is written as ${ }^{(2 S+1)} \Gamma$, where $\Gamma$ is a capital letter $(\mathrm{S}, \mathrm{P}, \mathrm{D}, \mathrm{F}, \mathrm{G} . .$.$) corresponding to$ the values of $L(0,1,2,3,4 \ldots)$. The multiplicity of a term, i.e., the number of micro states it regroups, is given by $(2 S+1) \times(2 L+1)$. An electronic configuration contains several terms and the sum of their multiplicities is equal to the degeneracy of the configuration. The procedure for finding them out is rather tedious, except for the ground term, for which Hund's rules make its determination easy. According to these rules (to be applied in the given order), the ground term has:

Rule 1 The largest spin multiplicity

Rule 2 The largest orbital multiplicity

For instance, for $\mathrm{Eu}^{\mathrm{III}}, 4 \mathrm{f}^{6}$, the largest multiplicity is obtained when each electron is associated with a unique $4 \mathrm{f}$ wavefunction: $\mathrm{S}=6 \times 1 / 2=3$; therefore, $(2 S+1)=7$. To obtain the largest orbital multiplicity, these electrons have to be related with wavefunctions having the largest $m_{\ell}$ values, i.e., $+3,+2,+1,0,-1$, and -2 ; the sum is 3 , henceforth $L=3$ and $\Gamma=\mathrm{F}$ : the ground term is a spin septet, ${ }^{7} \mathrm{~F}$ with overall multiplicity $7 \times 7=49$. 
The two movements of the electrons are in fact not independent, they couple and the strength of the interaction usually increases with the atomic number. To simplify the treatment of this interaction, Russel and Saunders have proposed to consider this coupling at the level of the overall angular momenta and not for each individual electron. This model is valid for lighter elements and not quite adequate for lanthanides for which an intermediate coupling scheme should be applied; however, it is in large use in view of its simplicity and we shall keep with it in this chapter. The Hamiltonian becomes:

$$
\mathcal{H}=\sum_{i=1}^{n}\left(-\frac{Z^{\prime}}{r_{i}}-\frac{1}{2} \Delta_{i}\right)+\sum_{i \neq j}^{n} \frac{1}{r_{i j}}+\lambda \cdot \vec{L} \cdot \vec{S} \quad \text { with } \quad \lambda= \pm \frac{\xi}{2 S}
$$

The spin-orbit coupling constant $\lambda$ is positive if the $4 \mathrm{f}$ subshell is less than half filled and negative if it is more than half filled. A new quantum number $J$, associated with the total angular momentum $\vec{J}=\vec{L}+\vec{S}$, has to be introduced with values ranging from $(L+S)$ to $(L-S)$. As a consequence, each term is further split into a number of spectroscopic levels ${ }^{(2 S+1)} \Gamma_{J}$ each with a $(2 J+1)$ multiplicity. Again, the sum of these multiplicities must be equal to the multiplicity of the term. For instance, the ground term of $\mathrm{Eu}^{\mathrm{III}}$ is split into ${ }^{7} \mathrm{~F}_{0},{ }^{7} \mathrm{~F}_{1},{ }^{7} \mathrm{~F}_{2},{ }^{7} \mathrm{~F}_{3},{ }^{7} \mathrm{~F}_{4},{ }^{7} \mathrm{~F}_{5}$, and ${ }^{7} \mathrm{~F}_{6}$ with multiplicities $1+3+5+7+9+11+13=49$. The ground level can be found with third Hund's rule:

Rule 3 if $n<(2 \ell+1), J=J_{\min } ; \quad$ if $n>(2 \ell+1), J=J_{\max }$.

Note that if the sub-shell is half filled, then $L=0$ and $J=S$. Additionally, $J$ may take half-integer values if $S$ is half-integer. The set of levels is referred to as a multiplet and this multiplet is a regular one if $n<(2 \ell+1)$, the energy of the levels increasing with increasing values of $J$, while it is inverted if $n>(2 \ell+1)$. This is illustrated with $\mathrm{Eu}^{\mathrm{III}}\left(4 \mathrm{f}^{6}\right)$ for which the ground level is ${ }^{7} \mathrm{~F}_{0}$ while it is ${ }^{7} \mathrm{~F}_{6}$ for $\mathrm{Tb}^{\mathrm{III}}$ $\left(4 \mathrm{f}^{8}\right)$. Finally, the energy difference between two consecutive spin-orbit levels with quantum numbers $J$ and $J^{\prime}=J+1$ is directly proportional to $J^{\prime}$ :

$$
\Delta E=\lambda \cdot J^{\prime}
$$

The electronic properties of the trivalent $4 \mathrm{f}$ free ions are summarized in Table 1.

\subsection{The Ions in a Ligand Field}

The above developments are valid for free ions. When a $\mathrm{Ln}^{\mathrm{III}}$ ion is inserted into a chemical environment, the spherical symmetry of its electronic structure is destroyed and the remaining $(2 J+1)$ degeneracy of its spectroscopic levels is partly lifted, depending on the exact symmetry of the metal-ion site. In view of the 
Table 1 Electronic properties of $\mathrm{Ln}^{\mathrm{III}}$ free ions

\begin{tabular}{|c|c|c|c|c|c|c|c|c|c|}
\hline$\overline{\mathrm{f}^{n}}$ & Multiplicity & No. of terms & No. of levels & Grou & d level & $\zeta / \mathrm{cm}^{-}$ & & $\lambda / \mathrm{cm}$ & $-1, \mathrm{a}$ \\
\hline$\overline{f^{0}} \quad f^{14}$ & 1 & 1 & 1 & ${ }^{1} \mathrm{~S}_{0}$ & ${ }^{1} \mathrm{~S}_{0}$ & - & - & - & - \\
\hline $\mathrm{f}^{1} \mathrm{f}^{13}$ & 14 & 1 & 2 & ${ }^{2} \mathrm{~F}_{5 / 2}$ & ${ }^{2} \mathrm{~F}_{7 / 2}$ & 625 & 2,870 & 625 & $-2,870$ \\
\hline$f^{2} \quad f^{12}$ & 91 & 7 & 13 & ${ }^{3} \mathrm{H}_{4}$ & ${ }^{3} \mathrm{H}_{6}$ & 740 & 2,628 & 370 & $-1,314$ \\
\hline$f^{3} \quad f^{11}$ & 364 & 17 & 41 & ${ }^{4} I_{9 / 2}$ & ${ }^{4} I_{15 / 2}$ & 884 & 2,380 & 295 & -793 \\
\hline$f^{4} \quad f^{10}$ & 1,001 & 47 & 107 & ${ }^{5} \mathrm{I}_{4}$ & ${ }^{5} \mathrm{I}_{8}$ & 1,000 & 2,141 & 250 & -535 \\
\hline$f^{5} \quad f^{9}$ & 2,002 & 73 & 198 & ${ }^{6} \mathrm{H}_{5 / 2}$ & ${ }^{6} \mathrm{H}_{15 / 2}$ & 1,157 & 1,932 & 231 & -386 \\
\hline$f^{6} \quad f^{8}$ & 3,003 & 119 & 295 & ${ }^{7} \mathrm{~F}_{0}$ & ${ }^{7} \mathrm{~F}_{6}$ & 1,326 & 1,709 & 221 & -285 \\
\hline $\mathrm{f}^{7}$ & 3,432 & 119 & 327 & ${ }^{8} \mathrm{~S}_{7 / 2}$ & & 1,450 & & 0 & \\
\hline
\end{tabular}

${ }^{\mathrm{a}}$ For aqua ions, except for $\mathrm{Ce}{ }^{\mathrm{III}}\left(\mathrm{Ce}: \mathrm{LaCl}_{3}\right)$ and $\mathrm{Yb}^{\mathrm{III}}\left(\mathrm{Yb}_{3} \mathrm{Ga}_{5} \mathrm{O}_{12}\right)$, from [2]. The first column refers to $\mathrm{f}^{1-7}$ and the second to $\mathrm{f}^{8-14}$

inner character of the $4 \mathrm{f}$ wavefunctions their mixing with the surrounding orbitals remains small and so is the resulting level splitting (a few hundreds of $\mathrm{cm}^{-1}$ ), so that this perturbation can be treated last. Nevertheless, the resulting Hamiltonian gets very complex, so that a simplifying concept has been put forward by $\mathrm{H}$. Bethe in 1929: the ligands are replaced by (negative) point charges generating a crystal (or ligand) electrostatic field which, in turn, interacts with the moving $4 \mathrm{f}$ electrons, generating a ligand-field (or crystal-field, or Stark) splitting of the spectroscopic levels.

With the parameterization introduced by B. G. Wybourne in 1965, the final Hamiltonian becomes:

$$
\mathcal{H}=\sum_{i=1}^{n}\left(-\frac{Z^{\prime}}{r_{i}}-\frac{1}{2} \Delta_{i}\right)+\sum_{i \neq j}^{n} \frac{1}{r_{i j}}+\lambda \cdot \vec{L} \cdot \vec{S}+\sum_{k, q, i} B_{q}^{k} C_{q}^{(k)}(i),
$$

where the summation involving $i$ is on all the $4 \mathrm{f}$ electrons, $B_{q}^{k}$ are ligand-field parameters, commonly treated as phenomenological parameters, and $C_{q}^{(k)}$ are components of tensor operators $\boldsymbol{C}^{(k)}$ which transform like the spherical harmonics used for the analytical form of the $4 \mathrm{f}$ wavefunctions. The running number $k$ must be even and smaller than $2 \ell$; for 4 f electrons it can, therefore, take values of $0,2,4$, and 6 . The values for $q$ are restricted by the point group of symmetry into which the $\mathrm{Ln}^{\mathrm{III}}$ ion is embedded, but in any case, $|q| \leq k$.

The $B_{q}^{k}$ parameters may be complex numbers but they have to be real for any symmetry group with a $180^{\circ}$ rotation about the $y$ axis or with the $x y$ plane being a mirror plane. The relationship between the 32 crystallographic symmetry groups and the $B_{q}^{k}$ parameters is given in Table 2. In order to compare the ligand field strengths in different compounds, F. Auzel has proposed the following expression for an "average" total ligand-field effect:

$$
N_{v}=\left[\frac{1}{4 \pi} \sum_{k, q} \frac{\left(B_{q}^{k}\right)^{2}}{(2 k+1)}\right]^{1 / 2} .
$$


Table 2 Non-zero crystal-field parameters for $\mathrm{f}^{n}$ electronic configurations and examples of corresponding crystal hosts [1]

\begin{tabular}{llll}
\hline Symmetry & Site symmetry & Crystal field parameters & Example \\
\hline Monoclinic & $C_{1}, C_{S}, C_{2}, C_{2 h}$, & $B_{0}^{2}, B_{0}^{4}, B_{0}^{6}, \Re\left(B_{2}^{2}\right), B_{2}^{4}, B_{2}^{6}, B_{4}^{4}, B_{4}^{6}, B_{6}^{6}$ & $\mathrm{LaF}_{3}$ \\
Rhombic & $C_{2 v}, D_{2}, D_{2 h}$ & $B_{0}^{2}, B_{0}^{4}, B_{0}^{6}, \Re\left(B_{2}^{2}, B_{2}^{4}, B_{2}^{6}, B_{4}^{4}, B_{4}^{6}, B_{6}^{6}\right)$ & $\mathrm{Y}_{3} \mathrm{Al}_{5} \mathrm{O}_{12}$ \\
Trigonal & $C_{3}, S_{6}$ & $B_{0}^{2}, B_{0}^{4}, B_{0}^{6}, \Re\left(B_{3}^{4}\right), B_{3}^{6}, B_{6}^{6}$ & $\mathrm{LiNbO}_{3}$ \\
& $C_{3 v}, D_{3}, D_{3 d}$ & $B_{0}^{2}, B_{0}^{4}, B_{0}^{6}, \Re\left(B_{3}^{4}, B_{3}^{6}, B_{6}^{6}\right)$ & $\mathrm{Y}_{2} \mathrm{O}_{2} \mathrm{~S}$ \\
Tetragonal & $C_{4}, S_{4}, C_{4 h}$ & $B_{0}^{2}, B_{0}^{4}, B_{0}^{6}, \Re\left(B_{4}^{4}\right), B_{4}^{6}$ & $\mathrm{LiYF}_{4}$ \\
& $C_{4 v}, D_{4}, D_{2 d}, D_{4 h}$ & $B_{0}^{2}, B_{0}^{4}, B_{0}^{6}, \Re\left(B_{4}^{4}, B_{4}^{6}\right)$ & $\mathrm{YPO}_{4}$ \\
Hexagonal & $C_{3 h}, D_{3 h}, C_{6}, C_{6 h}$, & $B_{0}^{2}, B_{0}^{4}, B_{0}^{6}, \Re\left(B_{6}^{6}\right)$ & $\mathrm{LaCl}_{3}$ \\
& $C_{6 v}, D_{6}, D_{6 h}$ & & \\
Cubic & $T, T_{d}, T_{h}, O, O_{h}$ & $B_{0}^{4}, B_{0}^{6}, \Re\left(B_{4}^{4}, B_{4}^{6}\right)^{\mathrm{a}}$ & $\mathrm{CeO}_{2}$ \\
\hline${ }^{\mathrm{a}} B_{4}^{4}=\frac{5}{\sqrt{70}} B_{0}^{4}$, & $B_{4}^{6}=-\sqrt{7 / 2} B_{0}^{6}$ & &
\end{tabular}

Finally, it is worth noting that electrostatic ligand-field effects do not completely lift the degeneracy of the $J$ levels of odd-numbered electronic configurations; in the latter case, all ligand-field sublevels are at least doubly degenerate (Kramer's doublets) and this degeneracy can only be removed by a magnetic field. A partial energy diagram, including crystal-field splittings is given in Fig. 2. Due to their large number, energy levels may extend up to $190,000 \mathrm{~cm}^{-1}$ for $n=6,7,8$, and are not yet fully explored, although an extension of Carnall's diagram up to this energy has been recently published [4].

The maximum numbers of ligand-field (or Stark) sublevels depend on the point group of symmetry: they are given in Table 3 versus values of $J$. This can be exploited for the determination of the symmetry point group from $\mathrm{f}-\mathrm{f}$ absorption or emission spectra, at least when $J$ is integer.

\section{Absorption Spectra}

Description of the interaction between photons (massless elemental particles of light) and matter considers the former behaving as waves comprised of two perpendicular fields, electric and magnetic, oscillating in time (henceforth the denomination of electromagnetic wave or radiation). When a photon is absorbed, its energy is transferred to an electron which then may be "pushed" into an orbital with higher energy. The absorption is promoted by "operators" linked to the nature of light: the odd-parity electric dipole (ED) operator $\vec{P}$, the even-parity magnetic dipole (MD) $\vec{M}$ and electric quadrupole (EQ) $\vec{Q}$ operators:

$$
\vec{P}=-e \sum_{i=1}^{n} \vec{r}_{i} \quad \vec{M}=-\frac{e h}{4 \pi m c} \sum_{i=1}^{n}\left(\vec{\ell}_{i}+2 \vec{s}_{i}\right) \quad \vec{Q}=\frac{1}{2} \sum_{i=1}^{n}\left(\vec{k} \cdot \vec{r}_{i}\right) \cdot \vec{r}_{i} .
$$

There are three types of electronic transitions involving lanthanide ions: sharp intraconfigurational $4 \mathrm{f}-4 \mathrm{f}$ transitions, broader $4 \mathrm{f}-5 \mathrm{~d}$ transitions, and broad 


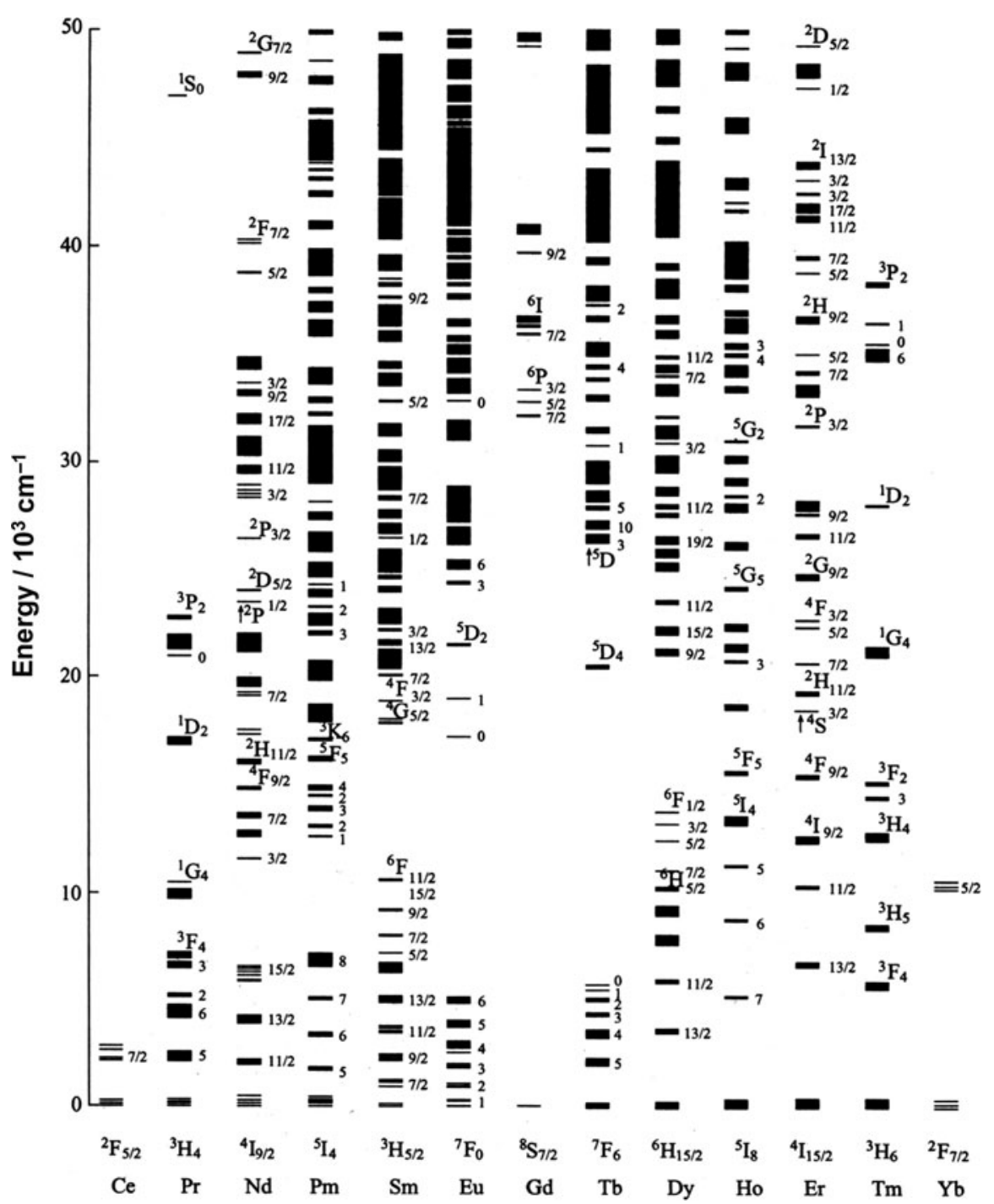

Fig. 2 Energy level diagram for $\mathrm{Ln}^{\mathrm{III}}$ ions doped in a low-symmetry crystal, $\mathrm{LaF}_{3}$. Redrawn from [3]

charge-transfer transitions (metal-to-ligand, MLCT or ligand-to-metal, LMCT). Not all transitions are permitted and the allowed ones are described by selection rules. Laporte's parity selection rule implies that states with the same parity cannot be connected by electric dipole transitions; as a consequence $\mathrm{f}-\mathrm{f}$ transitions are forbidden by the ED mechanism. However, when the lanthanide ion is under the influence of a ligand-field, non-centrosymmetric interactions allow the mixing of 
Table 3 Number of Stark levels versus the value of quantum number $J$

\begin{tabular}{lllllllllll}
\hline Symmetry & Site symmetry & \multicolumn{10}{c}{ Integer $J$} \\
\cline { 2 - 10 } & & 0 & 1 & 2 & 3 & 4 & 5 & 6 & 7 & 8 \\
\hline Cubic & $T, T_{d}, T_{h}, O, O_{h}$ & 1 & 1 & 2 & 3 & 4 & 4 & 6 & 6 & 7 \\
Hexagonal & $C_{3 h}, D_{3 h}, C_{6}, C_{6 h}, C_{6 v}, D_{6}, D_{6 h}$ & 1 & 2 & 3 & 5 & 6 & 7 & 9 & 10 & 11 \\
Trigonal & $C_{3}, S_{6} C_{3 v}, D_{3}, D_{3 d}$ & & & & & & & & & \\
Tetragonal & $C_{4}, S_{4}, C_{4 h}$, & 1 & 2 & 4 & 5 & 7 & 8 & 10 & 11 & 13 \\
& $C_{4 v}, D_{4}, D_{2 d}, D_{4 h}$ & & & & & & & & & \\
Low & $C_{1}, C_{S}, C_{2}, C_{2 h}, C_{2 v}, D_{2}, D_{2 h}$ & 1 & 3 & 5 & 7 & 9 & 11 & 13 & 15 & 17 \\
Symmetry & Site symmetry & & & & \multicolumn{7}{c}{ Half-integer $J$} \\
& $1 / 2$ & $3 / 2$ & $5 / 2$ & $7 / 2$ & $9 / 2$ & $11 / 2$ & $13 / 2$ & $15 / 2$ & $17 / 2$ \\
Cubic & $T, T_{d}, T_{h}, O, O_{h}$ & 1 & 1 & 2 & 3 & 3 & 4 & 5 & 6 & 6 \\
All others & See above & 1 & 2 & 3 & 4 & 5 & 6 & 7 & 8 & 9 \\
\hline
\end{tabular}

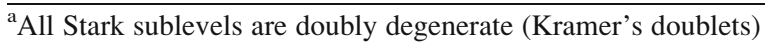

electronic states of opposite parity into the $4 \mathrm{f}$ wavefunctions, which somewhat relaxes the selection rules and the transition becomes partially allowed; it is called an induced (or forced) electric dipole transition. Magnetic dipole transitions are allowed, but their intensity is weak; in $4 \mathrm{f}-4 \mathrm{f}$ spectra, however, they often have intensity of the same order of magnitude as induced electric dipole transitions. Quadrupolar transitions are also parity allowed, but they are much weaker than MD transitions so that they are usually not observed. Some induced ED transitions are highly sensitive to minute changes in the $\mathrm{Ln}^{\mathrm{III}}$ environment and are called hypersensitive or sometimes pseudo-quadrupolar transitions because they apparently follow the selection rules of EQ transitions. A listing of experimentally identified such transitions is presented in Table 4; note, that these transitions are not necessarily the most intense ones in the optical spectra.

In addition to the parity selection rule, other rules are operative, for instance, on $\Delta S$ (spin selection rule, requiring no change of spin for all three mechanisms, $\Delta S=0), \Delta L$, and $\Delta J$; they will be detailed below. The selection rules are derived under several hypotheses which are not always completely fulfilled in reality (in particular 4f wavefunctions are not completely pure), so that the terms "forbidden" and "allowed" transitions are not accurate. Let's say that a forbidden transition has a low probability and an allowed transition a high probability of occurring.

\subsection{Induced ED $f-f$ Transitions: Judd-Ofelt Theory [5, 6]}

Judd-Ofelt (JO) theory has been established within the frame of the crystal-field concept and it provides a simple model for reproducing the intensities of $\mathrm{f}-\mathrm{f}$ transitions both in solids and solutions. It only takes into account the $4 \mathrm{f}^{n}$ electronic configuration, that is inter-configurational $4 \mathrm{f}^{n}-4 \mathrm{f}^{n-1} 5 \mathrm{~d}^{1}$ interactions are neglected. On the other hand, spin-orbit coupling is treated within the frame of the intermediate 
Table 4 Experimentally observed hypersensitive transitions for $\mathrm{Ln}^{\mathrm{III}}$ ions in optical spectra [5]. Energies/wavelengths are approximate

\begin{tabular}{llll}
\hline Ln & Transition & $\tilde{v} / \mathrm{cm}^{-1}$ & $\lambda / \mathrm{nm}$ \\
\hline $\mathrm{Pr}$ & 5,200 & 1,920 \\
$\mathrm{Nd}$ & ${ }^{3} \mathrm{~F}_{2} \leftarrow{ }^{3} \mathrm{H}_{4}$ & 17,300 & 578 \\
& ${ }^{4} \mathrm{G}_{5 / 2} \leftarrow{ }^{4} \mathrm{I}_{9 / 2}{ }^{\mathrm{a}}$ & 12,400 & 806 \\
& ${ }^{2} \mathrm{H}_{9 / 2},{ }^{4} \mathrm{~F}_{5 / 2} \leftarrow{ }^{4} \mathrm{I}_{9 / 2}$ & 19,200 & 521 \\
$\mathrm{Sm}$ & ${ }^{4} \mathrm{G}_{7 / 2},{ }^{3} \mathrm{~K}_{13 / 2} \leftarrow{ }^{4} \mathrm{I}_{9 / 2}$ & 6,400 & 1,560 \\
$\mathrm{Eu}$ & ${ }^{4} \mathrm{~F}_{1 / 2},{ }^{4} \mathrm{~F}_{3 / 2} \leftarrow{ }^{6} \mathrm{H}_{5 / 2}$ & 21,500 & 465 \\
& ${ }^{5} \mathrm{D}_{2} \leftarrow{ }^{7} \mathrm{~F}_{0}$ & 18,700 & 535 \\
& ${ }^{5} \mathrm{D}_{1} \leftarrow{ }^{7} \mathrm{~F}_{1}$ & 16,300 & 613 \\
$\mathrm{Gd}$ & ${ }^{5} \mathrm{D}_{0} \rightarrow{ }^{7} \mathrm{~F}_{2}$ & 32,500 & 308 \\
$\mathrm{~Tb}$ & ${ }^{6} \mathrm{P}_{5 / 2},{ }^{6} \mathrm{P}_{7 / 2} \leftarrow{ }^{8} \mathrm{~S}_{7 / 2}$ & - & - \\
$\mathrm{Dy}$ & ${ }^{\mathrm{b}}$ & 7,700 & 1,300 \\
& ${ }^{6} \mathrm{~F}_{11 / 2} \leftarrow{ }^{6} \mathrm{H}_{15 / 2}$ & 23,400 & 427 \\
$\mathrm{Ho}$ & ${ }^{4} \mathrm{G}_{11 / 2}, \mathrm{I}_{15 / 2} \leftarrow{ }^{6} \mathrm{H}_{15 / 2}$ & 27,700 & 361 \\
& ${ }^{3} \mathrm{H}_{6} \leftarrow{ }^{5} \mathrm{I}_{8}$ & 22,100 & 452 \\
$\mathrm{Er}$ & ${ }^{5} \mathrm{G}_{6} \leftarrow{ }^{5} \mathrm{I}_{8}$ & 26,400 & 379 \\
& ${ }^{4} \mathrm{G}_{11 / 2} \leftarrow{ }^{4} \mathrm{I}_{15 / 2}$ & 19,200 & 521 \\
$\mathrm{Tm}$ & ${ }^{2} \mathrm{H}_{11 / 2} \leftarrow{ }^{4} \mathrm{I}_{15 / 2}$ & 21,300 & 469 \\
& ${ }^{1} \mathrm{G}_{4} \leftarrow{ }^{3} \mathrm{H}_{6}$ & 12,700 & 787 \\
& ${ }^{3} \mathrm{H}_{4} \leftarrow{ }^{3} \mathrm{H}_{6}$ & 5,900 & 1,695 \\
\hline
\end{tabular}

${ }^{a}$ The transition ${ }^{4} \mathrm{G}_{5 / 2} \leftarrow{ }^{4} \mathrm{I}_{9 / 2}$ overlaps with ${ }^{2} \mathrm{G}_{7 / 2} \leftarrow{ }^{4} \mathrm{I}_{9 / 2}$

${ }^{b}$ None identified positively, but the ${ }^{5} \mathrm{D}_{4} \rightarrow{ }^{7} \mathrm{~F}_{5}$ transition shows sometimes ligand-induced pseudo-hypersensitivity

coupling scheme. The dipole strength in $\operatorname{esu}^{2} \mathrm{~cm}^{2}\left(=10^{36}\right.$ debye $\left.{ }^{2}\right)$ of an induced ED $\mathrm{f}-\mathrm{f}$ transition between states $\Psi$ and $\Psi^{\prime}$ is given by:

$$
D_{\mathrm{ED}}=e^{2} \sum_{\lambda=2,4,6} \Omega_{\lambda}\left|\left\langle\Psi\left\|U^{\lambda}\right\| \Psi^{\prime}\right\rangle\right|^{2}
$$

in which $e$ is the electric charge of the electron, wavefunctions $\Psi$ and $\Psi^{\prime}$ are full intermediate-coupled functions $\mathrm{f}^{n}[\mathrm{SL}] \mathrm{J}, U^{\lambda}$ are the irreducible tensor forms of the ED operator, and $\Omega_{\lambda}$ are the phenomenological JO parameters, expressed in $\mathrm{cm}^{2}$. The bracketed expressions in (12) are dimensionless doubly-reduced matrix elements which are tabulated (and insensitive to the metal-ion environment). Mathematical treatment of the parity mixing by the crystal-field perturbation leads to the selection rules for $\mathrm{f}-\mathrm{f}$ transitions reproduced in Table 5 .

JO parameters are adjustable parameters and they are calculated from the absorption spectrum $\varepsilon(\tilde{v})$. For an isotropic crystal or a solution, the experimental dipole strength is defined as:

$$
D(\exp )=\frac{10^{36}}{108.9 \cdot \tilde{v}_{\text {mean }} \cdot X_{\mathrm{A}}}\left((2 J+1) \cdot \frac{9 n}{\left(n^{2}+2\right)^{2}}\right) \int \varepsilon(\tilde{v}) \mathrm{d} \tilde{v}
$$


Table 5 Selection rules for intra-configurational $\mathrm{f}-\mathrm{f}$ transitions

\begin{tabular}{lllll}
\hline Operator & Parity & $\Delta S$ & $\Delta L$ & $\Delta J^{\mathrm{a}}$ \\
\hline ED & Opposite & 0 & $\leq 6$ & $\leq 6\left(2,4,6\right.$ if $J$ or $\left.J^{\prime}=0\right)$ \\
MD & Same & 0 & 0 & $0, \pm 1$ \\
EQ & Same & 0 & $0, \pm 1, \pm 2$ & $0, \pm 1, \pm 2$ \\
\hline
\end{tabular}

${ }^{\mathrm{a}} J=0$ to $J^{\prime}=0$ transitions are always forbidden

with $X_{\mathrm{A}}$ being the fractional population of the initial state while $\tilde{v}_{\text {mean }}$ is given by:

$$
\tilde{v}_{\text {mean }}=\frac{\int \tilde{v} \cdot \varepsilon(\tilde{v}) \mathrm{d} \tilde{v}}{\int \varepsilon(\tilde{v}) \mathrm{d} \tilde{v}} .
$$

The above equations assume that the absorption bands are symmetrical, i.e., either Gaussian or Lorentzian. If not, (13a) has to be replaced with:

$$
D(\exp )=\frac{10^{36}}{108.9 \cdot X_{\mathrm{A}}}\left((2 J+1) \frac{9 n}{\left(n^{2}+2\right)^{2}}\right) \int \frac{\varepsilon(\tilde{v})}{\tilde{v}} \mathrm{~d} \tilde{v} .
$$

Finally, $(2 J+1)$ is the degeneracy of the initial state and the expression involving the refractive index $n$ is known as Lorentz's local-field correction. Calculations of transition probabilities within the frame of JO theory are usually made assuming that all Stark sublevels within the ground level are equally populated and that the material under investigation is optically isotropic. The former hypothesis is only reasonable in some cases, e.g., when transitions initiate from non-degenerate states such as $\operatorname{Eu}\left({ }^{7} \mathrm{~F}_{0}\right)$. Otherwise, there is a Boltzmann distribution of the population among the crystal-field sublevels. The second assumption is not valid for uniaxial or biaxial crystals, but, of course, holds for solutions.

The phenomenological JO parameters are determined from a fit of (12) to the experimental values defined by (13a), using adequate matrix elements. The exact procedure is described in details in reference [6]. In the case of $\mathrm{Eu}^{\mathrm{III}}$ the procedure is quite simple since $\Omega_{2}, \Omega_{4}$, and $\Omega_{6}$ can be directly extracted from the dipole strength of the ${ }^{5} \mathrm{D}_{2} \leftarrow{ }^{7} \mathrm{~F}_{0},{ }^{5} \mathrm{D}_{4} \leftarrow{ }^{7} \mathrm{~F}_{0}$, and ${ }^{5} \mathrm{~L}_{6} \leftarrow{ }^{7} \mathrm{~F}_{0}$ transitions, respectively. An example is shown on Fig. 3 for europium tris(dipicolinate). Extensive tabulations of JO parameters can be found in reference [5] while spectra for all $\mathrm{Ln}^{\mathrm{III}}$ ions are presented in reference [8]; note that molar absorption coefficients are, with a few exceptions, smaller than $10 \mathrm{M}^{-1} \mathrm{~cm}^{-1}$ and very often smaller than 1 or even $0.1 \mathrm{M}^{-1} \mathrm{~cm}^{-1}$.

\section{$2.24 f-5 d$ and CT Transitions}

The promotion of a $4 \mathrm{f}$ electron into the $5 \mathrm{~d}$ sub-shell is parity allowed; the corresponding transitions are broader than $\mathrm{f}-\mathrm{f}$ transitions and their energy depends 


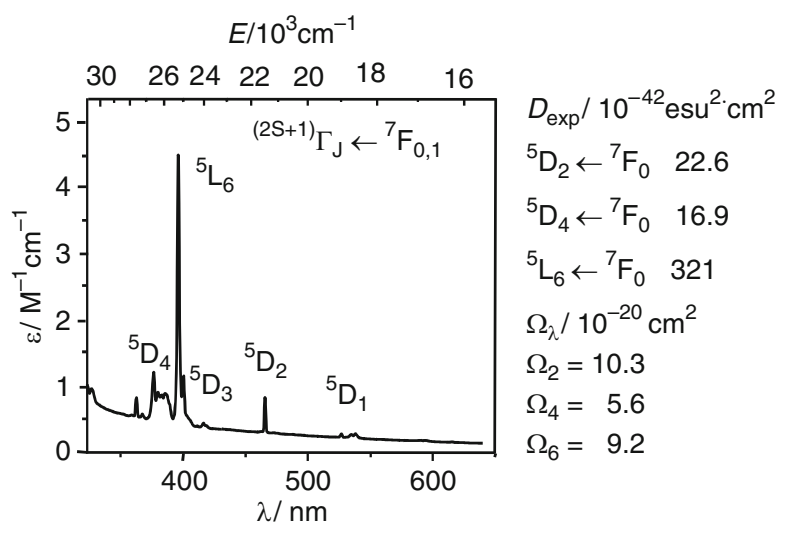

Fig. 3 Absorption spectrum of $\mathrm{Na}_{3}\left[\mathrm{Eu}(\mathrm{dpa})_{3}\right] 1.8 \times 10^{-2} \mathrm{M}$ in Tris- $\mathrm{HCl} 0.1 \mathrm{M}$ and associated dipole strengths and JO parameters. Redrawn from [7]

Fig. 4 Energy of the $4 f-5 d$ transitions in $\mathrm{Ln}^{\mathrm{III}}: \mathrm{CaF}_{2}$ (squares, [9]) and of the $2 \mathrm{p}$ (O)-4f LMCT transitions (triangles, [10])

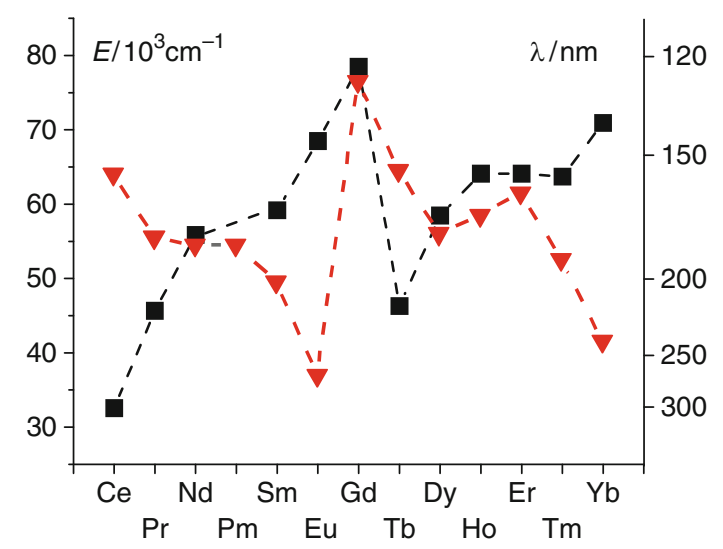

largely on the metal environment since the $5 \mathrm{~d}$ orbitals are external and interact directly with the ligand orbitals. The $4 \mathrm{f}-5 \mathrm{~d}$ transitions have high energies (Fig. 4) and only those of $\mathrm{Ce}^{\mathrm{III}}, \mathrm{Pr}^{\mathrm{III}}$, and $\mathrm{Tb}^{\mathrm{III}}$ are commonly observed. Figure 5 shows the crystal-field splitting of both the $4 \mathrm{f}^{1}\left({ }^{2} \mathrm{~F}_{5 / 2},{ }^{2} \mathrm{~F}_{7 / 2}\right)$ and $5 \mathrm{~d}^{1}\left({ }^{2} \mathrm{D}_{3 / 2},{ }^{2} \mathrm{D}_{5 / 2}\right)$ electronic configurations of $\mathrm{Ce}^{\mathrm{III}}$ in $D_{3 \mathrm{~h}}$ symmetry. In the spectrum displayed, the third transition to ${ }^{2} \mathrm{D}_{5 / 2}$ is not observed because it lies at too high energy. Conversely, the $\mathrm{Ce}^{\mathrm{III}}$ luminescence can be tuned from about 290 to $450 \mathrm{~nm}$, depending on the matrix into which the metal ion is inserted, because of large crystal-field effects on the $5 \mathrm{~d}^{1}$ excited configuration.

Charge-transfer transitions, both LMCT and MLCT, are allowed and have also high energies (Fig. 4), so that only the LMCT of Eu ${ }^{\mathrm{III}}$ and $\mathrm{Yb}^{\mathrm{III}}$ (possibly $\mathrm{Sm}^{\mathrm{III}}$ and $\mathrm{Tm}^{\mathrm{III}}$ ) are commonly observed in ordinary solvents, contrary to d-transition metal 

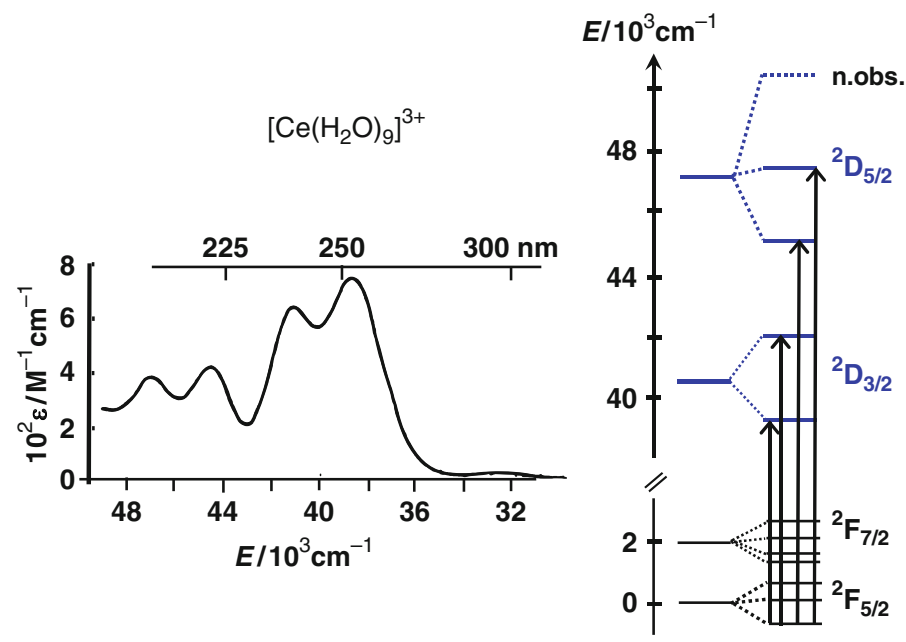

Fig. 5 Left: Absorption spectrum of $\left[\mathrm{Ce}\left(\mathrm{H}_{2} \mathrm{O}\right)_{9}\right]^{3+}$ and right: its assignment $\left(D_{3 \mathrm{~h}}\right.$ symmetry)

ions for which this type of transition is widespread. This is sometimes not well understood and the literature features many wrong assignments to MLCT transitions made by analogy to d-metal complexes.

\section{Emission Spectra}

With the exception of $\mathrm{La}^{\mathrm{III}}$ and $\mathrm{Lu}^{\mathrm{III}}$, all $\mathrm{Ln}^{\mathrm{III}}$ ions are luminescent and their $\mathrm{f}-\mathrm{f}$ emission lines cover the entire spectrum, from UV $\left(\mathrm{Gd}^{\mathrm{III}}\right)$ to visible (e.g., $\mathrm{Pr}^{\mathrm{III}}$, $\mathrm{Sm}^{\mathrm{III}}, \mathrm{Eu}^{\mathrm{III}}, \mathrm{Tb}^{\mathrm{III}}, \mathrm{Dy}^{\mathrm{III}}, \mathrm{Tm}^{\mathrm{III}}$ ) and near-infrared (NIR, e.g., $\mathrm{Pr}^{\mathrm{III}}, \mathrm{Nd}^{\mathrm{III}}, \mathrm{Ho}^{\mathrm{III}}, \mathrm{Er}^{\mathrm{III}}$, $\left.\mathrm{Yb}^{\mathrm{III}}[11]\right)$ ranges. Some ions are fluorescent $(\Delta S=0)$, others are phosphorescent $(\Delta S \neq 0)$, and some are both. The $\mathrm{f}-\mathrm{f}$ emission lines are sharp because the rearrangement consecutive to the promotion of an electron into a $4 \mathrm{f}$ orbital of higher energy does not perturb much the binding pattern in the molecules since $4 \mathrm{f}$ orbitals do not participate much in this binding (the covalency of a $\mathrm{Ln}^{\mathrm{III}}$-ligand bond is at most 5-7\%). Therefore, the internuclear distances remain almost the same in the excited state, which generates narrow bands and very small Stokes' shifts. A different situation is met in organic molecules for which excitation leads frequently to a lengthening of the chemical bonds, resulting in large Stokes' shifts and since the coupling with vibrations is strong, in broad emission bands (Fig. 6). The main emission lines observed in $\mathrm{Ln}^{\mathrm{III}}$ luminescence spectra are listed in Table 6, together with other key photophysical parameters.

As for absorption, emission of light through $\mathrm{f}-\mathrm{f}$ transitions is achieved by either electric dipole or magnetic dipole mechanisms, and the selection rules detailed in 
Fig. 6 Configurational coordinate diagram for emission from (left) an organic chromophore and (right) a lanthanide ion

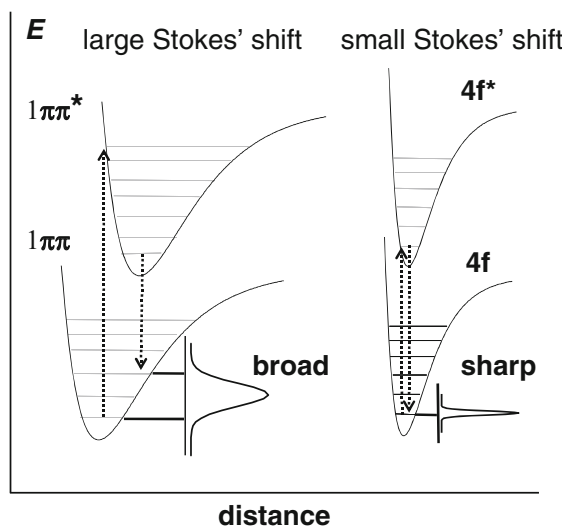

Table 5 apply. Important parameters characterizing the emission of light from a $\mathrm{Ln}^{\mathrm{III}}$ ion are the lifetime of the excited state $\tau_{\mathrm{obs}}=1 / k_{\mathrm{obs}}$ and the quantum yield $Q$. A general expression for the latter is simply

$$
Q=\frac{\text { number of emitted photons }}{\text { number of absorbed photons }} \text {. }
$$

The quantum yield is related to the rate at which the excited level is depopulated $k_{\mathrm{obs}}$ and to the radiative rate constant $k^{\mathrm{rad}}$ :

$$
Q_{\mathrm{Ln}}^{\mathrm{Ln}}=\frac{k^{\mathrm{rad}}}{k_{\mathrm{obs}}}=\frac{\tau_{\mathrm{obs}}}{\tau^{\mathrm{rad}}} .
$$

Subscript and superscript "Ln" have been added to avoid confusion with the other definition of quantum yield discussed below. The quantity defined in (16) is called the intrinsic quantum yield, that is, the quantum yield of the metal-centered luminescence upon direct excitation into the $4 \mathrm{f}$ levels. Its value reflects the extent of nonradiative deactivation processes occurring both in the inner- and outercoordination spheres of the metal ion. The rate constant $k_{\mathrm{obs}}$ is the sum of the rates of the various deactivation processes:

$$
k_{\mathrm{obs}}=k^{\mathrm{rad}}+\sum_{n} k_{n}^{\mathrm{nr}}=k^{\mathrm{rad}}+\sum_{i} k_{i}^{\mathrm{vibr}}(T)+\sum_{j} k_{j}^{\mathrm{pet}}(T)+\sum_{k} k_{k}^{\prime} \mathrm{nr},
$$

where $k^{\mathrm{rad}}$ and $k^{\mathrm{nr}}$ are the radiative and nonradiative rate constants, respectively; the superscript vibr points to vibration-induced processes while pet refers to photoinduced electron transfer processes such as those generated by LMCT states, for instance; the rate constants $k^{\prime}$ are associated with the remaining deactivation paths.

In absence of nonradiative deactivation processes, $k_{\mathrm{obs}}=k^{\mathrm{rad}}$ and the quantum yield would be equal to 1 , which is very rare. Examples are, in solid state and under 
Table 6 Ground (G), main emissive (I) and final (F) states for the most important $\mathrm{f}-\mathrm{f}$ emission bands, approximate corresponding wavelengths $(\lambda)$, energy gap between the emissive state and the highest SO level of the receiving state, and radiative lifetime of $\mathrm{Ln}^{\mathrm{III}}$ ions. More NIR lines are listed in [11]

\begin{tabular}{|c|c|c|c|c|c|c|}
\hline $\mathrm{Ln}$ & $\mathrm{G}$ & $\mathrm{I}$ & $\mathrm{F}$ & $\lambda / \mu \mathrm{m}$ or $\mathrm{nm}^{\mathrm{a}}$ & $\mathrm{Gap} / \mathrm{cm}^{-1, \mathrm{a}}$ & $\tau^{\mathrm{rad}} / \mathrm{ms}^{\mathrm{a}}$ \\
\hline $\mathrm{Ce}$ & ${ }^{2} \mathrm{~F}_{5 / 2}$ & $5 d$ & ${ }^{2} \mathrm{~F}_{5 / 2}$ & Tunable, $300-450$ & - & - \\
\hline \multirow[t]{3}{*}{$\operatorname{Pr}$} & ${ }^{3} \mathrm{H}_{4}$ & ${ }^{1} \mathrm{D}_{2}$ & ${ }^{3} \mathrm{~F}_{4},{ }^{1} \mathrm{G}_{4},{ }^{3} \mathrm{H}_{4},{ }^{3} \mathrm{H}_{5}$ & $1.0,1.44,600,690$ & 6,940 & $\left(0.05^{\mathrm{b}}-0.35\right)$ \\
\hline & & ${ }^{3} \mathrm{P}_{0}$ & ${ }^{3} \mathrm{H}_{J}(J=4-6)$ & $490,545,615,640$ & 3,910 & $\left(0.003^{\mathrm{b}}-0.02\right)$ \\
\hline & & ${ }^{3} \mathrm{P}_{0}$ & ${ }^{3} \mathrm{~F}_{J}(J=2-4)$ & 700,725 & & \\
\hline $\mathrm{Nd}$ & ${ }^{4} \mathrm{I}_{9 / 2}$ & ${ }^{4} \mathrm{~F}_{3 / 2}$ & ${ }^{4} \mathrm{I}_{J}(J=9 / 2-13 / 2)$ & $900,1.06,1.35$ & 5,400 & $0.42(0.2-0.5)$ \\
\hline \multirow[t]{3}{*}{$\mathrm{Sm}$} & ${ }^{6} \mathrm{H}_{5 / 2}$ & ${ }^{4} \mathrm{G}_{5 / 2}$ & ${ }^{6} \mathrm{H}_{J}(J=5 / 2-13 / 2)$ & $560,595,640,700,775$ & 7,400 & $6.26(4.3-6.3)$ \\
\hline & & ${ }^{4} \mathrm{G}_{5 / 2}$ & ${ }^{6} \mathrm{~F}_{J}(J=1 / 2-9 / 2)$ & $870,887,926,1.01,1.15$ & & \\
\hline & & ${ }^{4} \mathrm{G}_{5 / 2}$ & ${ }^{6} \mathrm{H}_{13 / 2}$ & 877 & & \\
\hline $\mathrm{Eu}^{\mathrm{c}}$ & ${ }^{7} \mathrm{~F}_{0}$ & ${ }^{5} \mathrm{D}_{0}$ & ${ }^{7} \mathrm{~F}_{J}(J=0-6)$ & $\begin{array}{l}580,590,615,650,720 \\
\quad 750,820\end{array}$ & 12,300 & $9.7(1-11)$ \\
\hline Gd & ${ }^{8} \mathrm{~S}_{7 / 2}$ & ${ }^{6} \mathrm{P}_{7 / 2}$ & ${ }^{8} \mathrm{~S}_{7 / 2}$ & 315 & 32,100 & 10.9 \\
\hline $\mathrm{Tb}$ & ${ }^{7} \mathrm{~F}_{6}$ & ${ }^{5} \mathrm{D}_{4}$ & ${ }^{7} \mathrm{~F}_{J}(J=6-0)$ & $\begin{array}{l}490,540,580,620,650 \\
\quad 660,675\end{array}$ & 14,800 & $9.0(1-9)$ \\
\hline \multirow[t]{2}{*}{ Dy } & ${ }^{6} \mathrm{H}_{15 / 2}$ & ${ }^{4} \mathrm{~F}_{9 / 2}$ & ${ }^{6} \mathrm{H}_{J}(J=15 / 2-9 / 2)$ & $475,570,660,750$ & 7,850 & $1.85(0.15-1.9)$ \\
\hline & & ${ }^{4} \mathrm{I}_{15 / 2}$ & ${ }^{6} \mathrm{H}_{J}(J=15 / 2-9 / 2)$ & $455,540,615,695$ & 1,000 & $3.22^{\mathrm{b}}$ \\
\hline \multirow[t]{3}{*}{$\mathrm{Ho}^{\mathrm{d}}$} & ${ }^{5} \mathrm{I}_{8}$ & ${ }^{5} \mathrm{~S}_{2}$ & ${ }^{5} \mathrm{I}_{J}(J=8,7)$ & 545,750 & 3,000 & $0.37\left(0.51^{\mathrm{b}}\right)$ \\
\hline & & ${ }^{5} \mathrm{~F}_{5}$ & ${ }^{5} \mathrm{I}_{8}$ & 650 & 2,200 & $0.8^{\mathrm{b}}$ \\
\hline & & ${ }^{5} \mathrm{~F}_{5}$ & ${ }^{5} \mathrm{I}_{7}$ & 965 & & \\
\hline \multirow[t]{4}{*}{$\mathrm{Er}^{\mathrm{e}}$} & ${ }^{4} \mathrm{I}_{15 / 2}$ & ${ }^{4} S_{3 / 2}$ & ${ }^{4} \mathrm{I}_{J}(J=15 / 2,13 / 2)$ & 545,850 & 3,100 & $0.7^{\mathrm{b}}$ \\
\hline & & ${ }^{4} \mathrm{~F}_{9 / 2}$ & ${ }^{4} \mathrm{I}_{15 / 2}$ & 660 & 2,850 & $0.6^{\mathrm{b}}$ \\
\hline & & ${ }^{4} \mathrm{I}_{9 / 2}$ & ${ }^{4} \mathrm{I}_{15 / 2}$ & 810 & 2,150 & $4.5^{\mathrm{b}}$ \\
\hline & & ${ }^{4} \mathrm{I}_{13 / 2}$ & ${ }^{4} \mathrm{I}_{15 / 2}$ & 1.54 & 6,500 & $0.66(0.7-12)$ \\
\hline \multirow[t]{3}{*}{$\mathrm{Tm}$} & ${ }^{3} \mathrm{H}_{6}$ & ${ }^{1} \mathrm{D}_{2}$ & ${ }^{3} \mathrm{~F}_{4},{ }^{3} \mathrm{H}_{4},{ }^{3} \mathrm{~F}_{3},{ }^{3} \mathrm{~F}_{2}$ & $450,650,740,775$ & 6,650 & 0.09 \\
\hline & & ${ }^{1} \mathrm{G}_{4}$ & ${ }^{3} \mathrm{H}_{6},{ }^{3} \mathrm{~F}_{4},{ }^{3} \mathrm{H}_{5}$ & $470,650,770$ & 6,250 & 1.29 \\
\hline & & ${ }^{3} \mathrm{H}_{4}$ & ${ }^{3} \mathrm{H}_{6}$ & 800 & 4,300 & $3.6^{\mathrm{b}}$ \\
\hline $\mathrm{Yb}$ & ${ }^{2} \mathrm{~F}_{7 / 2}$ & ${ }^{2} \mathrm{~F}_{5 / 2}$ & ${ }^{2} \mathrm{~F}_{7 / 2}$ & 980 & 10,250 & 1.3 or $2.0^{f}$ \\
\hline
\end{tabular}

${ }^{a}$ Values for the aqua ions [8], otherwise stated, and ranges of observed lifetimes in all media, if available, between parentheses

${ }^{b}$ Doped in $\mathrm{Y}_{2} \mathrm{O}_{3}$ or in $\mathrm{YLiF}_{4}(\mathrm{Ho})$, or in $\mathrm{YAl}_{3}\left(\mathrm{BO}_{3}\right)_{4}$ (Dy)

${ }^{\mathrm{c}}$ Luminescence from ${ }^{5} \mathrm{D}_{1},{ }^{5} \mathrm{D}_{2}$, and ${ }^{5} \mathrm{D}_{3}$ is sometimes observed as well

${ }^{\mathrm{d}}$ The laser transition ${ }^{5} \mathrm{I}_{7} \rightarrow{ }^{5} \mathrm{I}_{8}(2.1-2.2 \mu \mathrm{m})$ is used in medical surgery of the eyes

${ }^{\mathrm{e}}$ Luminescence from four other states has also been observed: ${ }^{4} \mathrm{D}_{5 / 2},{ }^{2} \mathrm{P}_{3 / 2},{ }^{4} \mathrm{G}_{11 / 2},{ }^{2} \mathrm{H}_{9 / 2}$

${ }^{\mathrm{f}}$ Complexes in solution: $1.2-1.3 \mathrm{~ms}$; solid-state inorganic compounds: $\approx 2 \mathrm{~ms}$

excitation at $254 \mathrm{~nm}, \mathrm{Y}_{2} \mathrm{O}_{3}:$ Eu (5\%) with $Q=0.99$ and terbium benzoate with $Q=1$ [12]; in solution, a terbium complex with a dipyrazoylpyridine bearing aminocarboxylate coordinating groups was reported having $Q=0.95$ [13].

Temperature-dependent vibrational deactivation processes can often be fitted to an Arrhenius-type of equation [14]:

$$
\ln \left(k_{\mathrm{obs}}-k_{0}\right)=\ln A-\frac{E_{\mathrm{A}}}{R T},
$$

where $k_{0}$ is the rate constant at $0 \mathrm{~K}$ (practically: at $4 \mathrm{~K}$, or even at $77 \mathrm{~K}$ ) which allows one to decipher which vibration is responsible for it; examples are presented in references [15] and [16]. 
The intrinsic quantum yield essentially depends on the energy gap $\Delta E$ between the emissive state of the metal ion and the highest sublevel of its ground, or receiving, multiplet. The smaller this gap, the easier is its closing by nonradiative deactivation processes, for instance, through vibrations of bound ligands, particularly those with high energy such as $\mathrm{O}-\mathrm{H}, \mathrm{N}-\mathrm{H}$, or $\mathrm{C}-\mathrm{H}$. With the assumption that the deactivating phonons involved have all the same energy $\hbar \omega$, the temperaturedependent rate constant $k^{\mathrm{vibr}}(T)$ for the quenching of a single excited level is described by the following expression [2]:

$$
k^{\mathrm{vibr}}(T)=k^{\mathrm{vibr}}(0)\left(1-e^{-\hbar \omega / k_{B} T}\right)^{-i} \text { with } i=\frac{\Delta E}{\hbar \omega},
$$

where $k_{B}$ is Boltzmann's constant $\left(1.38 \times 10^{-23} \mathrm{~J} \mathrm{~K}^{-1} \approx 0.695 \mathrm{~cm}^{-1}\right), i$ the number of phonons required to bridge the gap, and $k^{\mathrm{vibr}}(0)$ the spontaneous rate at $0 \mathrm{~K}$. The latter heavily depends on the order $n$ of the process. In practice, the excited level possesses several crystal-field sublevels, the population of which is in thermal equilibrium. This equilibrium is reached in times short compared to the multiphonon decay time, but since phonon-induced decay rates are significantly slower for the upper levels in view of the larger energy gaps, depopulation of the lower crystal field sublevel is the major contribution to the deactivation process. A rule of thumb is that radiative de-excitation will compete efficiently with multi-phonon processes if the energy gap is more than 6 quanta of the most energetic vibration present in the molecule. This type of nonradiative deactivation is especially detrimental to NIR luminescence [11]: for $\mathrm{Er}^{\mathrm{III}}$, for instance, a C-H vibrator located outside the inner coordination sphere at a distance between 20 and $30 \AA$ from the emitting center induces a radiationless rate equal to the radiative one.

Determination of the intrinsic quantum yield with (16) requires evaluation of the radiative lifetime which is related to Einstein's rates of spontaneous emission $A$ from an initial state $\left|\Psi_{J}\right\rangle$, characterized by a quantum number $J$, to a final state $\left|\Psi_{J^{\prime}}^{\prime}\right\rangle$ :

$$
A\left(\Psi_{J}, \Psi_{J^{\prime}}^{\prime}\right)=k^{\mathrm{rad}}=\frac{1}{\tau^{\mathrm{rad}}}=\frac{64 \pi^{4} \tilde{v}^{3}}{3 h(2 J+1)}\left[\frac{n\left(n^{2}+2\right)^{2}}{9} D_{\mathrm{ED}}+n^{3} D_{\mathrm{MD}}\right],
$$

where $\tilde{v}$ is the mean energy of the transition defined in (13b), $h$ is Planck's constant, $n$ is the refractive index; $D_{\mathrm{ED}}$ is given by (12) and $D_{\mathrm{MD}}$ by (21):

$$
D_{\mathrm{MD}}=\left(\frac{e h}{4 \pi m_{e} c}\right)^{2}\left|\left\langle\Psi|| \mathrm{L}+2 \mathrm{~S} \| \Psi^{\prime}\right\rangle\right|^{2} .
$$

The bracketed matrix elements are tabulated and the radiative lifetime can, therefore, be extracted from the spectral intensity, that is from (12), (20), and (21). Except in few cases, this calculation is not trivial and large errors may occur, including those pertaining to the hypotheses made within JO theory. 
In particular, it has been assumed that the emitting and receiving levels are $(2 J+1)$-fold degenerate or, if split by crystal field effects, that all the sublevels are equally populated. This is obviously not true and in the case of $\mathrm{Er}^{\mathrm{III}}$ this may lead to up to $20 \%$ errors. Even larger errors have been found for $\mathrm{Ln}^{\mathrm{III}}$ tris(dipicolinate) [7].

On the other hand, if the absorption spectrum corresponding to an emission spectrum is known, which may be the case when the luminescence transitions terminate onto the ground level, the radiative lifetime can be simply calculated from the following equation where $N_{\mathrm{A}}$ is Avogadro's number $\left(6.023 \times 10^{23}\right)$ :

$$
\frac{1}{\tau^{\mathrm{rad}}}=2303 \times \frac{8 \pi c n^{2} \tilde{v}^{2}(2 J+1)}{N_{\mathrm{A}}\left(2 J^{\prime}+1\right)} \int \varepsilon(\tilde{v}) \mathrm{d} \tilde{v} .
$$

In the special case of Eu ${ }^{\text {III }}$ for which one transition $\left({ }^{5} \mathrm{D}_{0} \rightarrow{ }^{7} \mathrm{~F}_{1}\right)$ has pure magnetic origin, a convenient simplified equation can be derived [17]:

$$
A\left(\Psi_{J}, \Psi_{J^{\prime}}^{\prime}\right)=\frac{1}{\tau^{\mathrm{rad}}}=A_{\mathrm{MD}, 0} \cdot n^{3}\left(\frac{I_{\mathrm{tot}}}{I_{\mathrm{MD}}}\right)
$$

with $A_{\mathrm{MD}, 0}$ being a constant equal to $14.65 \mathrm{~s}^{-1}$ and $\left(I_{\mathrm{tot}} / I_{\mathrm{MD}}\right)$ the ratio of the total integrated emission from the $\mathrm{Eu}\left({ }^{5} \mathrm{D}_{0}\right)$ level to the ${ }^{7} \mathrm{~F}_{J}$ manifold $(J=0-6)$ to the integrated intensity of the MD transition ${ }^{5} \mathrm{D}_{0} \rightarrow{ }^{7} \mathrm{~F}_{1}$.

An example of radiative lifetime calculations based on the various procedures evoked above can be found in reference [7], the results of which are collected in Table 7. The limits of JO approach are clearly seen both for $\mathrm{Eu}(\mathrm{error} \approx-20 \%)$ and $\mathrm{Tb}$ (error $>-100 \%)$. On the other hand, (22) and (23) yield trustworthy results: a radiative lifetime of $1.2 \mathrm{~ms}$ has been reported for [ $\mathrm{Yb}(\mathrm{dtpa})]^{2-}[17]$.

Finally, there are two important points to be stressed here in order to correct many errors reported in the literature. Firstly, the radiative lifetime is characteristic of one emitting state. If several excited states of an $\mathrm{Ln}^{\mathrm{III}}$ ion emit light, then each of

Table 7 Experimental intrinsic quantum yields, observed and radiative lifetimes of $\mathrm{M}_{3}\left[\operatorname{Ln}(\mathrm{dpa})_{3}\right]$ $(\mathrm{M}=\mathrm{Na}$ or $\mathrm{Cs})$ samples in solution $(0.1 \mathrm{M}$ Tris- $\mathrm{HCl}, \mathrm{pH}$ 7.4) and solid state at $295 \mathrm{~K} ; 2 \sigma$ are given between parentheses [7]

\begin{tabular}{llllll}
\hline Sample & $Q_{L n}^{L n} / \%$ & $\tau_{\text {obs }} / \mathrm{ms}$ & \multicolumn{3}{c}{$\tau^{\mathrm{rad}} / \mathrm{ms}^{\mathrm{a}}$} \\
\cline { 4 - 6 } & & & (i) & (ii) & (iii) \\
\hline Eu, $1.8-3.7 \times 10^{-2} \mathrm{M}$ & $41(2)$ & $1.7(0.1)$ & $4.1(3)$ & 3.15 & 4.0 \\
Eu, solid state & $68(4)$ & $1.8(0.1)$ & $2.6(2)$ & - & 2.7 \\
$\mathrm{~Tb}, 2.0 \times 10^{-2} \mathrm{M}$ & $-^{\mathrm{c}}$ & $1.74(1)$ & - & 1.0 & - \\
$\mathrm{Tb}$, solid state & $72(5)$ & $1.36(2)$ & $1.9(1)$ & - & - \\
$\mathrm{Yb}, 4.04 \times 10^{-2} \mathrm{M}$ & $-^{\mathrm{c}}$ & $2.23(1)^{\mathrm{d}}$ & - & - & $1.31(2)$ \\
\hline
\end{tabular}

${ }^{a}$ Successively: (i) experimental, i.e., from (16), (ii) from JO theory (20), (iii) from (23) for Eu and (22) for $\mathrm{Yb}$

${ }^{\mathrm{b}}$ Refractive index $=1.517$

${ }^{\mathrm{c}}$ Determination not feasible

${ }^{\mathrm{d}}$ In $\mu \mathrm{s}$ 
them will have a characteristic radiative lifetime. Moreover, the radiative lifetime is not a constant for a given ion and a given electronic level. Indeed, there is a dependence on the refractive index, as clearly shown in (20), so that transposition of a literature value to a specific compound cannot be made directly, which explains the wide range of $\tau^{\text {rad }}$ values reported for an individual $\mathrm{Ln}^{\mathrm{III}}$ ion (Table 6).

\section{Sensitization of Lanthanide Luminescence}

Since the dipole strengths of $\mathrm{f}-\mathrm{f}$ transitions are very small, direct excitation into the 4f excited levels rarely yields highly luminescent materials, even if the intrinsic quantum yield is large, unless considerable excitation power is used (laser excitation, given the sharpness of the absorption bands). Therefore, an alternative path has been worked out which is called luminescence sensitization or antenna effect. The luminescent ion is imbedded into a matrix or an organic environment such that the latter is a good light harvester. Energy is then transferred from the excited surroundings onto the metal ion which eventually gives off its characteristic light. Note, that several of the $\mathrm{Ln}^{\mathrm{III}}$ excited states may be implied in this process. The use of charge transfer or 4f5d states for collecting and transferring energy has long been well established in inorganic phosphors for lighting applications. On the other hand, the tuning of the electronic properties of organic ligands to achieve the same goal starts only to be understood since the process is more involved in view of the numerous electronic levels and mechanisms which may be implied.

Here, we focus on the latter case for which efficient light-harvesting is mainly performed by the aromatic $\left(\pi \rightarrow \pi^{*}\right)$ and/or $\left(n \rightarrow \pi^{*}\right)$ transitions of unsaturated ligands displaying large cross sections for one-photon absorption. Alternatively, singlet states, intra-ligand charge transfer states (ILCT), ligand-to-metal charge transfer states (LMCT), or ${ }^{3}$ MLCT states localized on a transition-metal containing ligand may also play this role [18]. As a result of the poor expansion of the $4 \mathrm{f}$ orbitals, the Ln-ligand bonds are mainly electrostatic and only some minute mixing of metal and ligand electronic wavefunctions contributes to covalency. It, therefore, appears justified to consider separately ligand-centered and metal-centered excited states in lanthanide complexes, and a Jablonsky diagram is adequate for representing energy migration paths (Fig. 7). In this diagram, grey arrows representing energy transfer to the metal ion do not point to a specific excited state since several of them may intervene.

One of the main energy migration path implies Laporte- and spin-allowed ligand-centered absorptions followed by intersystem crossing $\left({ }^{1} \mathrm{~S}^{*} \rightarrow{ }^{3} \mathrm{~T}^{*}, k_{\text {ISC }}\right)$ reaching the long-lived ligand-centered triplet state, from which ${ }^{3} \mathrm{~T}^{*} \rightarrow \operatorname{Ln}^{*}\left(k^{\mathrm{et}}\right)$ energy transfer occurs. Spontaneous metal-centered radiative emission completes the light-conversion process. It is to be stressed that although important, this energy transfer path is by far not the only operative one. Kleinerman who studied over 600 lanthanide chelates pointed out as early as 1969 that excited singlet states may 


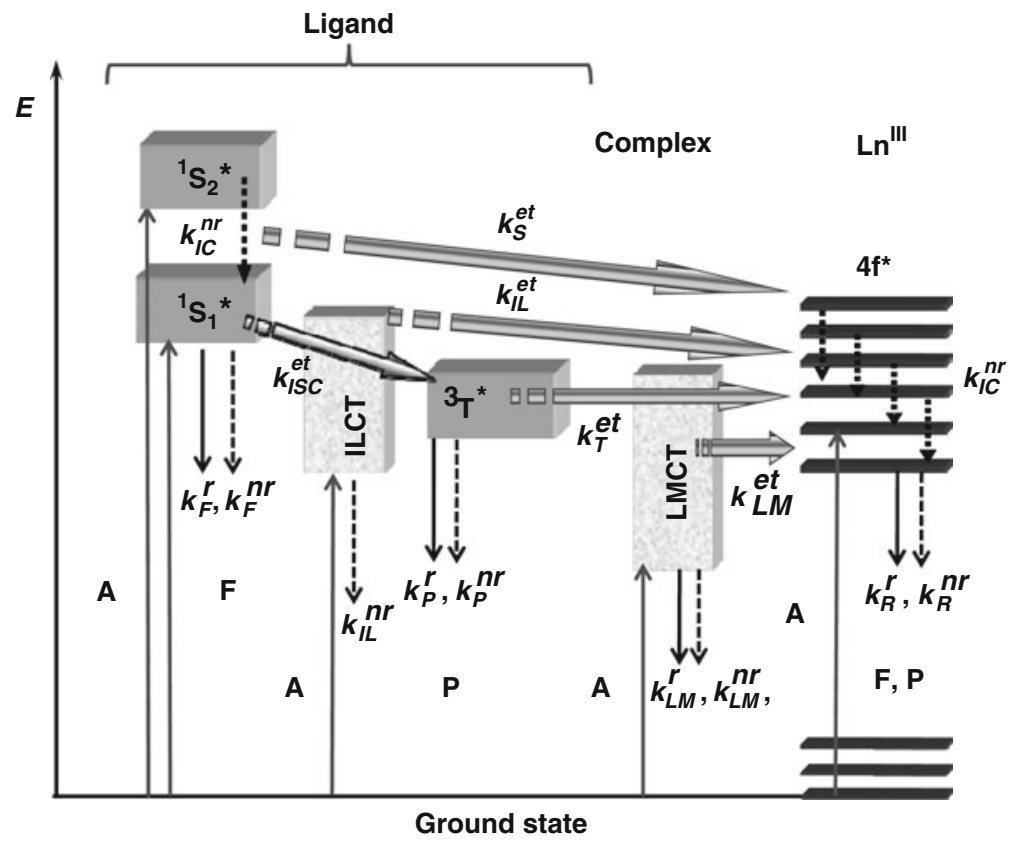

Fig. 7 Schematic representation of energy absorption, migration, emission (plain arrows) and dissipation (dotted arrows) processes in a lanthanide complex. ${ }^{1} \mathrm{~S}^{*}$ or $\mathrm{S}=$ singlet state, ${ }^{3} \mathrm{~T}^{*}$ or $\mathrm{T}=$ triplet state, $\mathrm{A}=$ absorption, $\mathrm{F}=$ fluorescence, $\mathrm{P}=$ phosphorescence, $k=$ rate constant, $r=$ radiative, $n r=$ nonradiative, $\mathrm{IC}=$ internal conversion, $\mathrm{ISC}=$ intersystem crossing, ILCT $($ or IL) $=$ intra-ligand charge transfer, LMCT $($ or LM) $=$ ligand-to-metal charge transfer. Back transfer processes are not drawn for the sake of clarity

contribute to the transfer and may even be the privileged donor states, depending on the relative values of the rate constants for the various intervening processes [19]. Since triplet states are quenched by oxygen, an easy way of establishing their involvement in the energy transfer process is to bubble oxygen into the solution: a reduction in $\mathrm{Ln}^{\mathrm{III}}$ luminescence proves their role but no effect may simply means $k^{\text {et }}>k^{\mathrm{O} 2}$. In fact a workable model of the entire energy-converting mechanism has shown that considering as many as 20-30 rate constants (including those describing back transfers) may be necessary [20]. All these potential energy funnels, particularly if ILCT, LMCT, as well as ${ }^{3}$ MLCT states are included, render difficult the a priori precise design of highly luminescent lanthanide-containing edifices.

Once the ligand is excited, subsequent intramolecular energy migrations obey Fermi's golden rule governing resonant energy transfer (24), whereby $W_{\mathrm{DA}}$ is the probability of energy transfer, $\Omega_{\mathrm{DA}}$ is the spectral overlap integral between the absorption spectrum of the acceptor $\mathrm{A}$ and the emission spectrum of the donor $\mathrm{D}$, while $\mathcal{H}^{\prime}$ is the perturbation operator in the matrix element $\left\langle\mathrm{D}^{*} \mathrm{~A}\left|\mathcal{H}^{\prime}\right| \mathrm{DA}^{*}>\right.$.

$$
W_{\mathrm{DA}}=\left(4 \pi^{2} / h\right) \cdot\left|<\mathrm{D}^{*} \mathrm{~A}\right| \mathcal{H}^{\prime}\left|\mathrm{DA}^{*}>\right|^{2} \cdot \Omega_{\mathrm{DA}} .
$$




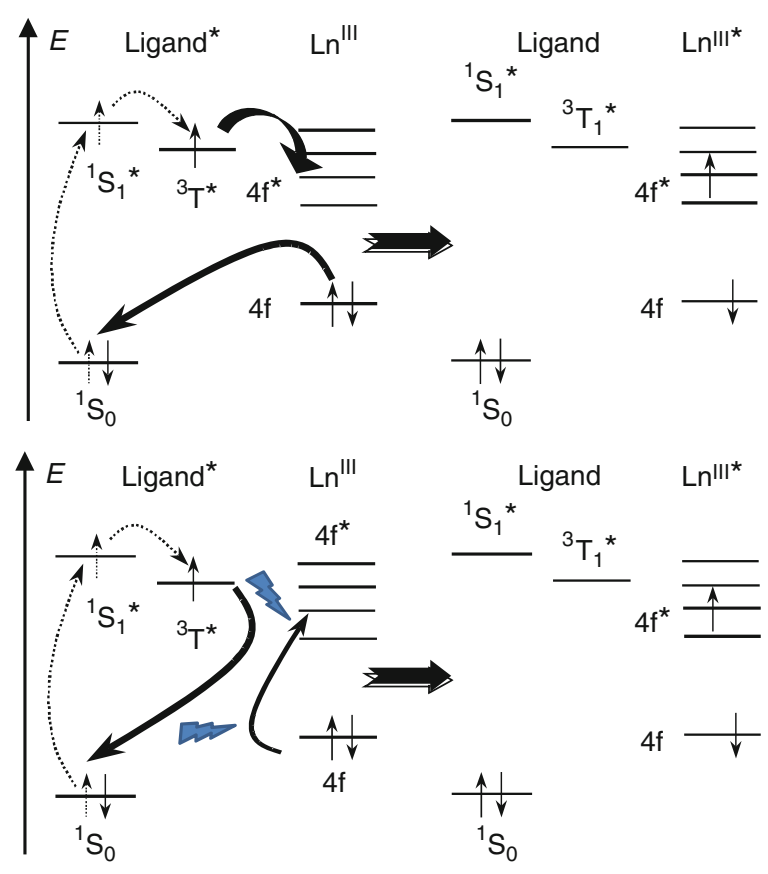

Fig. 8 Dexter (top) and Förster (bottom) energy transfer mechanisms

Depending on the electromagnetic nature of $\mathcal{H}^{\prime}$, a double-electron exchange (Dexter) mechanism or an electrostatic multipolar (Förster) mechanism have been proposed and theoretically modeled. They are sketched on Fig. 8 for the simple ${ }^{1} \mathrm{~S}^{*}{ }^{3} \mathrm{~T}{ }^{*}-\mathrm{Ln}^{*}$ path. Their specific dependences on the distance $d$ separating the donor $\mathrm{D}$ from the acceptor A, i.e., $e^{-\beta d}$ for double-electron exchange and $d^{-6}$ for dipole-dipolar processes, respectively, often limit Dexter mechanism to operate at short distance (typically $30-50 \mathrm{pm}$ ) at which orbital overlap is significant, while Förster mechanism may extend over much longer distances (up to 1,000 pm).

In addition, a $d^{-8}$-dependent dipole-quadrupolar mechanism may also be quite effective at short to medium-range distances; in fact, depending on $\Omega_{\mathrm{DA}}$, it may be as efficient as the dipole-dipole mechanism up to distances as long as $300 \mathrm{pm}$.

For lanthanides possessing low-lying charge-transfer excited states (e.g., Eu ${ }^{\text {III }}$, $\mathrm{Sm}^{\mathrm{III}}$ ) or for complexes having low-lying ILCT states, the energy transfer process is further affected by additional nonradiative quenching arising from back energy transfer onto the ligand (not shown on Fig. 7). Since in this case the accepting states are quite broad, minute differences in their energy may lead to large differences in the spectral overlap, and therefore, in the overall quantum yield. $\mathrm{Yb}^{\mathrm{III}}$ represents a special case since it has only one, low-lying, excited state $\left({ }^{2} \mathrm{~F}_{5 / 2}\right)$ and several excitation mechanisms have been proposed [11].

For the molecular edifices discussed here, another definition of quantum yield ought to be made: the overall quantum yield, that is the quantum yield of 
the metal-centered luminescence upon ligand excitation. It is related to the intrinsic quantum yield by the following equation:

$$
Q_{\mathrm{L}}^{\mathrm{Ln}}=\eta_{\text {pop }}^{\mathrm{D}} \eta_{\mathrm{et}} Q_{\mathrm{Ln}}^{\mathrm{Ln}}=\eta_{\mathrm{sens}} Q_{\mathrm{Ln}}^{\mathrm{Ln}}
$$

The three components in the middle term represent (1) the efficiency $\eta_{\text {pop }}^{\mathrm{D}}$ with which the feeding level $\left({ }^{3} \mathrm{~T}\right.$, ILCT, LMCT, ${ }^{3} \mathrm{MLCT}$, possibly a $4 \mathrm{f} 5 \mathrm{~d}$ state) is populated by the initially excited state (the corresponding rate constant is $k_{\text {ISC }}^{\text {et }}$ if ${ }^{1} \mathrm{~S}^{*}$ is excited and ${ }^{3} \mathrm{~T} *$ is the donor level, see Fig. 7), (2) the efficiency of the energy transfer $\left(\eta_{\mathrm{et}}\right)$ from the donor state to the accepting $\mathrm{Ln}^{\mathrm{III}}$ ion, and (3) the intrinsic quantum yield. The overall sensitization efficiency, $\eta_{\text {sens }}$ can be accessed experimentally if both the overall and intrinsic quantum yields are known or, alternatively, the overall quantum yield and the observed and radiative lifetimes:

$$
\eta_{\text {sens }}=\frac{Q_{\mathrm{L}}^{\mathrm{Ln}}}{Q_{\mathrm{Ln}}^{\mathrm{Ln}}}=Q_{\mathrm{L}}^{\mathrm{Ln}} \frac{\tau^{\mathrm{rad}}}{\tau_{\mathrm{obs}}} .
$$

The lifetime method is especially easy to implement for $\mathrm{Eu}^{\mathrm{III}}$ compounds since the radiative lifetime is readily determined from the emission spectrum via (23). Some data are reported in Table 8.

An important remark at this stage is that the intrinsic quantum yield is directly proportional to $\tau_{\text {obs }}$, but not necessarily the overall quantum yield since a change in

Table 8 Quantum yields, observed and radiative lifetimes, as well as sensitization efficiency for $\mathrm{Eu}^{\mathrm{III}}$ tris(dipicolinate) and bimetallic Eu ${ }^{\mathrm{III}}$ helicates; all data are at room temperature, for solutions in Tris- $\mathrm{HCl} 0.1 \mathrm{M}(\mathrm{pH}=7.4) ; 2 \sigma$ are given between parentheses [7, 21]. See Fig. 9 for formulae

\begin{tabular}{llllll}
\hline Sample & $Q_{L}^{E u}$ & $Q_{E u}^{E u}$ & $\tau_{\text {obs }} / \mathrm{ms}$ & $\tau^{\mathrm{rad}} / \mathrm{ms}$ & $\eta_{\text {sens }}$ \\
\hline$\left[\mathrm{Eu}(\mathrm{dpa})_{3}\right]^{3-}$ & $0.29(2)$ & $0.41(2)$ & $1.7(1)$ & $4.1(3)$ & $0.76(6)$ \\
{$\left[\mathrm{Eu}_{2}\left(\mathrm{~L}^{\mathrm{Cl}}\right)_{3}\right]$} & $0.24(2)$ & $0.37(4)$ & $2.4(1)$ & $6.8(3)$ & $0.67(10)$ \\
{$\left[\mathrm{Eu}_{2}\left(\mathrm{~L}^{\mathrm{C} 2}\right)_{3}\right]$} & $0.21(2)$ & $0.37(4)$ & $2.4(1)$ & $6.9(3)$ & $0.58(8)$ \\
{$\left[\mathrm{Eu}_{2}\left(\mathrm{~L}^{\mathrm{C} 3}\right)_{3}\right]$} & $0.11(2)$ & $0.36(4)$ & $2.2(1)$ & $6.2(3)$ & $0.30(5)$ \\
\hline
\end{tabular}
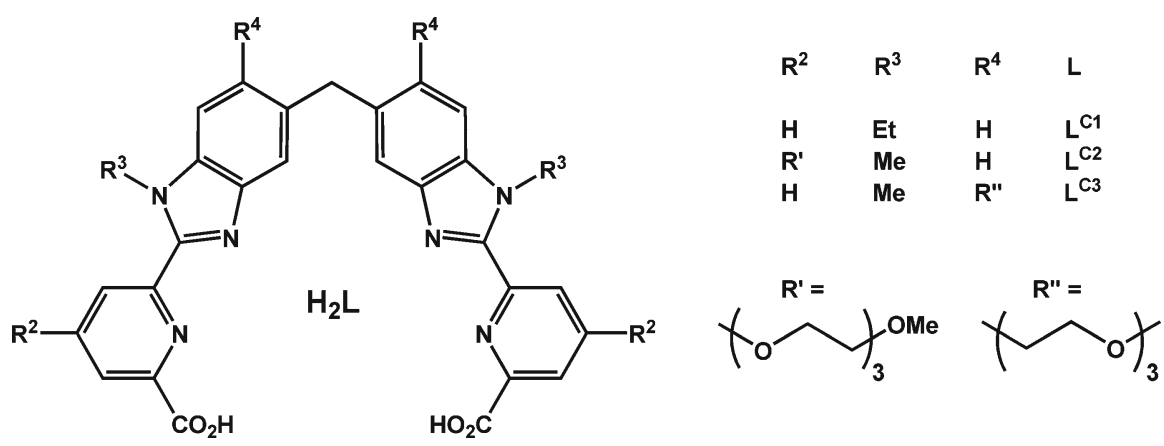

Fig. 9 Chemical structures of the ligands mentioned in Table 8 
the inner coordination sphere inducing, for instance, a smaller nonradiative deactivation rate may also influence $\eta_{\text {sens }}$ (in one way or the other) through the resulting electronic changes in the molecular edifice. Another point is that the distinction between intrinsic and overall quantum yields is often unclear in the literature, particularly for NIR-emitting ions for which direct experimental determination of the quantum yield is rarely performed. Authors commonly rely on (16) to evaluate the intrinsic quantum yield from lifetime determination and from a "literature" value of $\tau^{\text {rad }}$, so that extreme caution must be exercised in discussing these estimates [11].

\subsection{Design of Efficient Lanthanide Luminescent Bioprobes}

The ligand design for building efficient lanthanide luminescent bioprobes (LLBs) must meet several requirements, both chemical, photophysical, and biochemical: (1) efficient sensitization of the metal luminescence, (2) embedding of the emitting ion into a rigid and protective cavity minimizing nonradiative deactivation, (3) long excited state lifetime, (4) water solubility, (5) large thermodynamic stability, (6) kinetic inertness, (7) intense absorption above $330 \mathrm{~nm}$, and (8) whenever relevant, ability to couple to bioactive molecules while retaining their photophysical properties and not altering the bio-affinity of the host.

From the chemical point of view, it is best when the coordination sphere is saturated, i.e., when 8-10 donor atoms are bound to the metal ion, and when the coordinating groups are strong since in vivo experiments require large pLns (defined as $-\log \left[\mathrm{Ln}^{\mathrm{III}}\right]_{\text {free }}$ in water, at $\mathrm{pH} 7.4,\left[\mathrm{Ln}^{\mathrm{III}}\right]_{\mathrm{t}}=1 \mu \mathrm{M}$, and [Ligand $]_{\mathrm{t}}=$ $10 \mu \mathrm{M}$; ideally pLn should be $>20$ ). Carboxylates, aminocarboxylates, phosphonates, hydroxyquinolinates, and hydroxypyridinones are good candidates, while $\beta$ diketonates which have excellent photophysical properties have the tendency to be less stable. In aqueous solutions, the enthalpy and entropy changes upon complex formation between $\mathrm{Ln}^{\mathrm{III}}$ cations and many ionic ligands are predominantly influenced by changes in hydration of both the cation and the ligand(s). Complexation results in a decrease in hydration, yielding positive entropy changes favorable to the complexation process. On the other hand, dehydration is endothermic and contribution from bond formation between the cation and the ligand(s) often does not compensate this unfavorable energy contribution to the variation in Gibbs free energy, so that the overall complexation process is generally entropy driven. Therefore, it is advantageous to resort to polydentate ligands for building a coordination environment around $\mathrm{Ln}^{\mathrm{III}}$ ions. Macrocyclic complexes based on the cyclen framework [22] or on cryptands [23] are also proved to be quite adequate, as well as self-assembled mono- and bi-nuclear triple helical edifices [21].

The photophysical requirements are related to the two aspects described in (25): energy transfer $\left(\eta_{\text {sens }}\right)$ and minimization of nonradiative processes $\left(Q_{\mathrm{Ln}}^{\mathrm{Ln}}\right)$. The first one is difficult to master in view of the intricate processes going on (Fig. 7, [20]). Some authors have nevertheless tried to establish phenomenological rules. One has, however, to be cautious in applying them since these rules rely on a rather simple 
and naive picture: the ${ }^{1} \mathrm{~S}^{*}{ }^{3} \mathrm{~T}^{*}-\mathrm{Ln} *$ energy transfer path on one hand, and the consideration that the only parameter of importance is the energy gap between ${ }^{3} \mathrm{~T}^{*}$ and the emitting $\mathrm{Ln}^{\mathrm{III}}$ level. Some of the relationships are exemplified on Fig. 10 and examination of these data clearly point to the difficulty in establishing a dependable relationship. The following lessons can be drawn from these data:

- The maximum values of the quantum yields usually occur when the triplet state energy is close to the energy of one of the higher excited states of the metal ion, consistent with the fact that the emissive level is usually not directly fed by the ligand excited states (except maybe in the case of the Schiff base complexes depicted at the bottom of Fig. 10). When the energy of the feeding state becomes closer to the energy of the emitting state, back-energy transfer operates and the quantum yield goes down: this is true for both $\mathrm{Eu}^{\mathrm{III}}$ and $\mathrm{Tb}^{\mathrm{III}}$ and a "safe" energy difference minimizing this process is around $2,500-3,000 \mathrm{~cm}^{-1}$.

- Inspection of the $\mathrm{Eu}^{\mathrm{III}}$ quantum yields clearly demonstrates that the energy of the triplet state corresponding to the larger values depends on the type of ligand: it is close to the ${ }^{5} \mathrm{D}_{0}$ level for Schiff base complexes, to the ${ }^{5} \mathrm{D}_{1}$ level for $\beta$-diketonates, and to the ${ }^{5} \mathrm{D}_{2}$ level for polyaminocarboxylates.

- For the two series of $\mathrm{Eu}^{\mathrm{III}}$ and $\mathrm{Tb}^{\mathrm{III}}$ complexes with the same polyaminocarboxylate ligands, the maximum values reached by the quantum yield of $\mathrm{Tb}^{\mathrm{III}}$ are larger than those of $\mathrm{Eu}^{\mathrm{III}}$ : this reflects the smaller $\mathrm{Eu}\left({ }^{5} \mathrm{D}_{0}{ }^{-}{ }^{7} \mathrm{~F}_{6}\right)$ energy gap compared to the $\mathrm{Tb}\left({ }^{5} \mathrm{D}_{4}-{ }^{7} \mathrm{~F}_{0}\right)$ gap (Table 6).

It has been shown for calixarenes that more efficient ISC transfers take place when the energy difference between the singlet and triplet states is around $5,000 \mathrm{~cm}^{-1}$; therefore, ligand designers try to keep to the following phenomenological rules: $\Delta E\left({ }^{1} \mathrm{~S}^{*}-^{3} \mathrm{~T}^{*}\right) \approx 5,000 \mathrm{~cm}^{-1}$ and $\Delta E\left({ }^{3} \mathrm{~T}^{*}-\mathrm{Ln}{ }^{*}\right.$ emissive level $)$ in the range 2,500-3,500 $\mathrm{cm}^{-1}$. These are, however, golden rules only and sometimes minute energy differences in the ligand states lead to large differences in overlap between the emission spectrum of the donor and the absorption spectrum of the acceptor, resulting in large differences in quantum yield [27].

The second aspect, namely minimization of nonradiative deactivation has two facets: avoiding low-lying LMCT states, essentially for $\mathrm{Sm}^{\mathrm{III}}, \mathrm{Eu}^{\mathrm{III}}$, and $\mathrm{Yb}^{\mathrm{III}}$, and avoiding high energy vibrations in the first and second coordination spheres; the latter aspect is dealt with in Sect. 5.4.

\subsection{Practical Measurements of Absolute Quantum Yields}

Quantum yield measurements are simple in their principle, but very difficult to carry out experimentally, particularly when it comes to the luminescence of lanthanide ions and to intrinsic quantum yield, $\mathrm{f}-\mathrm{f}$ absorptions being faint. There are two main methods: the comparative method in which the sample under examination is compared to a standard with known quantum yield, and the absolute method which 

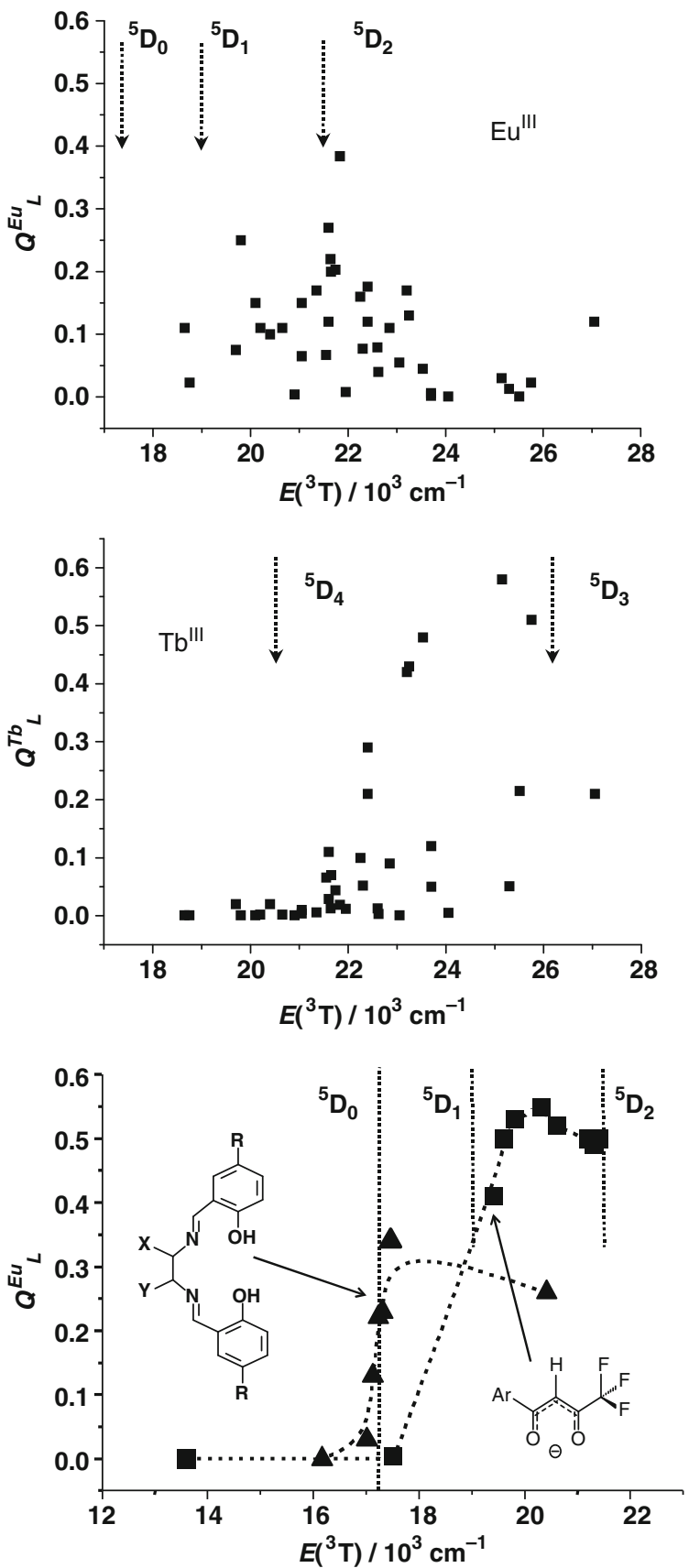

Fig. 10 Relationships between the triplet state energy of the feeding ligand and the quantum yields of $39 \mathrm{Eu}^{\mathrm{III}}$ (top) and $\mathrm{Tb}^{\mathrm{III}}$ (middle) polyaminocarboxylates [24] and of $\mathrm{Eu}^{\mathrm{III}}$ Schiff bases (triangles, [25]) and $\beta$-diketonates (squares, [26]) 
determines the amount of absorbed and emitted light with an integrating sphere since luminescence is emitted in all directions.

In the comparative method, the quantum yield of the unknown sample (indices $\mathrm{x}$ ) is given relative to the quantum yield of the standard (indices $S$ ) by:

$$
Q_{\mathrm{L}}^{\mathrm{Ln}}=Q_{\mathrm{S}} \times \frac{E_{x}}{E_{\mathrm{S}}} \times \frac{A_{\mathrm{S}}\left(\lambda_{\mathrm{S}}\right)}{A_{x}\left(\lambda_{x}\right)} \times \frac{I_{\mathrm{S}}\left(\lambda_{\mathrm{S}}\right)}{I_{x}\left(\lambda_{x}\right)} \times \frac{n_{x}{ }^{2}}{n_{\mathrm{S}}{ }^{2}},
$$

with $E$ being the integrated and corrected emission spectrum, $A$ the absorbance at the excitation wavelength $\lambda, I$ the intensity of the excitation source at the excitation wavelength, and $n$ the refractive index. In principle, linearity between the intensity of the emitted light and the concentration of the sample is only achieved if $A<0.05$, so that samples should be diluted to reach this value of absorbance, while being cautious not to dissociate the complex. In practice, if both the standard and the sample are excited at the same wavelength $\lambda_{\text {exc }}$ (a highly desirable, although not always achievable situation), measurements can be safely carried out up to $A=0.5$ since the inner-filter effect would be the same for both samples. In this case, (27a) simplifies to:

$$
Q_{\mathrm{L}}^{\mathrm{Ln}}=Q_{\mathrm{S}} \times \frac{E_{x}}{E_{\mathrm{S}}} \times \frac{A_{\mathrm{S}}\left(\lambda_{\mathrm{exc}}\right)}{A_{x}\left(\lambda_{\mathrm{exc}}\right)} \times \frac{n_{x}^{2}}{n_{\mathrm{S}}^{2}},
$$

or to $(27 \mathrm{c})$ if the standard and the unknown sample are in the same solvent:

$$
Q_{\mathrm{L}}^{\mathrm{Ln}}=Q_{\mathrm{S}} \times \frac{E_{x}}{E_{\mathrm{S}}} \times \frac{A_{\mathrm{S}}\left(\lambda_{\mathrm{exc}}\right)}{A_{x}\left(\lambda_{\mathrm{exc}}\right)} .
$$

In case $A_{x} \neq A_{S}$ and at least one of them is $>0.05$, then the absorbances in (27a-c) should be replaced by:

$$
A \rightarrow\left(1-10^{-A}\right)
$$

to take into account the different inner-filter effects. The correction is small for small differences in absorbances but can become very important: e.g., if $A_{S}=0.1$ and $A_{x}=0.15,0.2,0.4$, and 0.6 , respectively, then the corrected $A_{S} / A_{x}$ ratios would be $0.42,1.79,2.93$, and 3.64 instead of $0.5,2,4$ and 6 , respectively, corresponding to corrections of $-5,-10,-19,-27$, and $-39 \%$, respectively.

It is also essential that emission spectra are corrected for the instrumental function established with a standard calibrated lamp. It is wise not to use the calibration curve given by the manufacturer of the spectrometer and to re-measure this instrumental function at regular intervals because many items influence it, particularly the emission intensity of the excitation lamp and the quantum efficiency of the detector (which both decrease with time). In case (27a) is used, the excitation instrumental function has to be known as well. Regarding the standard, it is best when its emission spectrum overlaps the emission spectrum of the unknown 
Table 9 Selected useful standards for quantum yield determinations at room temperature. More extensive listings can be found in [28, 29]

\begin{tabular}{|c|c|c|c|}
\hline Compound $^{\mathrm{a}}$ & conc./solvent ${ }^{\mathrm{b}}$ & Range (nm) & $Q$ \\
\hline Quinine sulfate & aq. $\mathrm{H}_{2} \mathrm{SO}_{4}(0.5 \mathrm{M})$ & $400-600$ & 0.546 \\
\hline Cresyl violet & Methanol & $600-650$ & $0.54(3)$ \\
\hline $\mathrm{Cs}_{3}\left[\mathrm{~Tb}(\mathrm{dpa})_{3}\right]$ & $6.5 \times 10^{-5} \mathrm{M}$, Tris- $\mathrm{HCl} 0.1 \mathrm{M}$ & $480-670$ & $0.22(2)$ \\
\hline \multirow[t]{2}{*}[\mathrm{Ru}(\mathrm{bpy})_{3}]{$\left(\mathrm{ClO}_{4}\right)_{3}$} & $10^{-5} \mathrm{M}$, aerated water & $550-800$ & $0.028(2)$ \\
\hline & $10^{-5} \mathrm{M}$, de-aerated water & & $0.043(2)$ \\
\hline $\mathrm{Cs}_{3}\left[\mathrm{Eu}(\mathrm{dpa})_{3}\right]$ & $7.5 \times 10^{-5} \mathrm{M}$, Tris- $\mathrm{HCl} 0.1 \mathrm{M}$ & $580-690$ & $0.24(2)$ \\
\hline$\left[\mathrm{Yb}(\mathrm{tta})_{3}\left(\mathrm{H}_{2} \mathrm{O}\right)_{2}\right]$ & $10^{-3} \mathrm{M}$, toluene & $950-1,080$ & 0.0035 \\
\hline
\end{tabular}

${ }^{\mathrm{a}}$ bpy = bipyridine; tta $=$ thenoyltrifluoroacetylacetonate

${ }^{\mathrm{b}}$ When not given, the concentration should be such that $A<0.1$ (usually $c<10^{-5} \mathrm{M}$ )

sample; a safe way to proceed is to use two different standards and to measure them against each other as well. Each measurement should also be repeated several times. Selected standards useful in lanthanide photophysics are listed in Table 9.

The absolute method using an integration sphere has been in use for a long time, particularly by physicists or scientists involved in the design of phosphor materials [30]. A specially designed integration sphere has been produced in our laboratory which fairly well fulfills the needs of chemists and biochemists. It has a small 2inch diameter to ensure maximum sensitivity and is manufactured in Zenith ${ }^{\circledR}$ Teflon [7]. Samples are put in 2.4-mm I.D. quartz capillaries, themselves inserted into a protective quartz tube. The modified de Mello et al. method [30] requires the measurement of (1) $L_{a}$, the integrated intensity of light exiting the sphere when the empty capillary is illuminated at the excitation wavelength (Rayleigh scattering band); (2) $L_{c}$, the same integrated intensity at the excitation wavelength when the sample is introduced into the sphere; these two measurements often necessitate the use of attenuators (transmission $0.01-10 \%$ ); (3) $E_{c}$ the integrated intensity of the entire emission spectrum. The absolute quantum yield is then given by:

$$
Q_{\text {abs }}=\frac{E_{c}}{\left[L_{a}\left(\lambda_{\text {exc }}\right)-L_{c}\left(\lambda_{\text {exc }}\right)\right] F_{\text {att }}\left(\lambda_{\text {exc }}\right)},
$$

whereby $F_{\text {att }}\left(\lambda_{\text {exc }}\right)$ is the correction for the attenuators used. Reproducible and accurate data can be obtained when the fraction of absorbed light $\alpha=\left(L_{a}-L_{c}\right) / L_{a}$ is in the range 0.10-0.90 [7]; both solid state samples and solutions (minimum volume: $60 \mu \mathrm{L}$ ) can be measured. This method also requires carefully established instrumental functions; it is illustrated in Fig. 11.

\section{Information Extracted from Lanthanide Luminescent Probes}

The most important applications of lanthanide luminescent stains used as structural or analytical probes are summarized below. Any luminescent $\mathrm{Ln}^{\mathrm{III}}$ may act as a luminescent probe, but some ions either bear more information or are more 

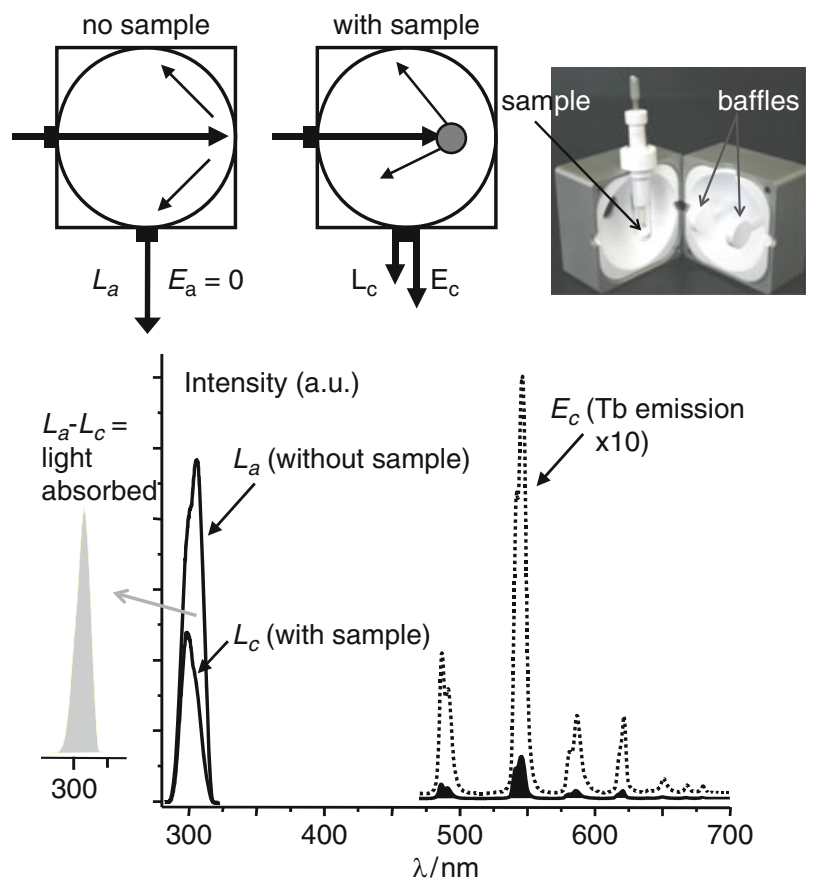

Fig. 11 Top: Integration sphere and bottom: example of quantum yield determination on a $\mathrm{Tb}^{\mathrm{III}}$ sample (this work)

luminescent than others, so that they are preferentially used. This is the case of $\mathrm{Eu}^{\mathrm{III}}$, which will be extensively referred to in the following description [31].

\subsection{Metal Ion Sites: Number, Composition, and Population Analysis}

Lanthanide ions have been used as substitutes for $\mathrm{Ca}^{\mathrm{II}}$ and $\mathrm{Zn}^{\mathrm{II}}$ in proteins to obtain information on the number of metallic sites (by simple titration) and on their composition. This may of course be extended to any molecule or materials. One very useful transition in this respect is the highly forbidden and faint Eu $\left({ }^{5} \mathrm{D}_{0} \rightarrow{ }^{7} \mathrm{~F}_{0}\right)$ transition which is best detected in excitation mode by analyzing the emission of the hypersensitive transition ${ }^{5} \mathrm{D}_{0} \rightarrow{ }^{7} \mathrm{~F}_{2}$; since both the emitting and end states are non-degenerate, its number of components indicates the number of different metal-ion sites. Moreover, the energy of this transition depends on the nephelauxetic effect $\delta_{\mathrm{i}}$ generated by coordinated atoms and ions; at $298 \mathrm{~K}$ :

$$
\tilde{v}_{\text {calc }}=17,374+C_{\mathrm{CN}} \sum_{i=1}^{C N} n_{i} \delta_{\mathrm{i}}
$$


with $C_{\mathrm{CN}}$ being a constant depending on the coordination number and $n_{\mathrm{i}}$ the number of coordinating groups with nephelauxetic effect $\delta_{\mathrm{i}}$. The latter are tabulated for the most common ligands [32] and some predictions are rather accurate [21], which allows one to check the composition of the inner coordination sphere.

When several metal ion sites are present in a compound, population analysis can be carried out in two main ways. For Eu ${ }^{\mathrm{III}}$, since the intensity of the MD transition ${ }^{5} \mathrm{D}_{0} \rightarrow{ }^{7} \mathrm{~F}_{1}$ is independent of the metal-ion environment, a spectral decomposition of the transition recorded under broad band excitation into its components measured under selective laser excitation, followed by integration yields the population $P_{i}$ of each site [31]. More generally, one can rely on lifetime measurement, since the luminescence decay will be a multi-exponential function which may be analyzed, for instance, with Origin ${ }^{\circledR}$, using the following equations:

$$
\begin{gathered}
I(t)=A+I_{0} \sum_{i=1}^{n} B_{i} e^{-k_{i} t}, \\
P_{i}=\frac{B_{i} / k_{i}}{\sum_{i=1}^{n}\left(B_{i} / k_{i}\right)} .
\end{gathered}
$$

In recording the decay, one has to make sure that (1) there is no artifact at the beginning of the decay (remaining light from the light pulse), (2) the decay is recorded during at least 5-6 lifetimes, (3) the signal at the end goes back to the background value, and (4) the decay is defined by a sufficient number of data points. Even if experimental data are of high quality, it is difficult to determine populations smaller than $5 \%$ and to decompose decays with more than 2 or 3 exponential functions or when the two lifetimes are either very different or quite similar. The example given on Fig. 12 illustrates a bi-exponential analysis of an $\operatorname{Eu}\left({ }^{5} \mathrm{D}_{0}\right)$ decay.

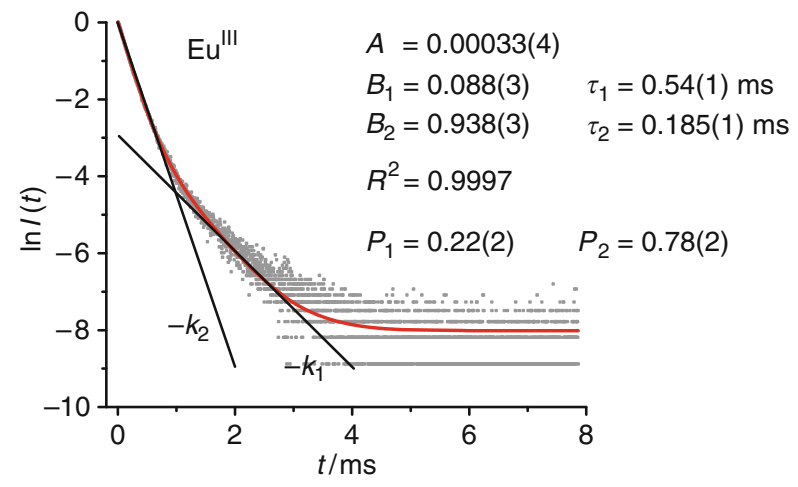

Fig. 12 Luminescence decay for an Eu ${ }^{\mathrm{III}}$ sample with its bi-exponential analysis; straight lines correspond to the two decay rates and the red line is the calculated fit (this work) 


\subsection{Site Symmetry Through Crystal-Field Analysis}

This aspect is related to the Stark splitting of the levels described in Table 3. Again here, Eu ${ }^{\text {III }}$ luminescence is the easiest to analyze given the non-degeneracy of the emissive ${ }^{5} \mathrm{D}_{0}$ level. When allied to high-resolution selective laser excitation of components of the ${ }^{5} \mathrm{D}_{0} \leftarrow{ }^{7} \mathrm{~F}_{0}$ transition, symmetry of multi-site molecules and materials can be worked out easily, based on group-theoretical considerations [31, 33]. Such detailed analyses are not discussed here.

On the other hand, the $\operatorname{Eu}\left({ }^{5} \mathrm{D}_{0} \rightarrow{ }^{7} \mathrm{~F}_{1}\right)$ transition represents an interesting case. A simple examination of its splitting tells immediately at which crystal system the compound belongs: cubic if only 1 component is seen, axial (i.e., hexagonal, tetragonal, or trigonal, labeled $\mathrm{A}$ and $\mathrm{E}$ in group-theoretical notation) if there are two components, and low symmetry if the maximum splitting of three appears. For truly low-symmetry species, the three components are equally spaced and tend to have the same intensity. However, when the coordination sphere is close to an idealized higher symmetry, the splitting is unsymmetrical. In this case, three important pieces of information can be extracted for symmetries close to axial symmetry: (1) the sign of the $\mathrm{B}_{2}^{0}$ crystal-field parameter which depends on the relative energetic position of the $A$ and $E$ sublevels of ${ }^{7} F_{1},(2)$ its value thanks to a phenomenological relationship between $\Delta E(\mathrm{~A}-\mathrm{E})$ and this parameter [34], and (3) the extent of the deviation from the idealized symmetry given by the splitting of the E sublevel. In the example depicted on Fig. 13, the crystal field parameter has a value of $\mathrm{ca}-600 \mathrm{~cm}^{-1}$ and the coordination polyhedron $\mathrm{EuN}_{6} \mathrm{O}_{3}$ appears to be only slightly distorted from the idealized $D_{3 h}$ symmetry with $\Delta E(\mathrm{E}-\mathrm{E})$ equal to $31 \mathrm{~cm}^{-1}$.

\subsection{Strength of Metal-Ligand Bonds: Vibronic Satellite Analysis}

The analysis depicted above requires high-resolution spectra. It is sometimes complicated by the occurrence of vibronic satellites which may artificially increase the number of components of a given transition, so again care has to be exercised. Vibronic transitions have the tendency to be strongest when associated with

Fig. 13 Left: Ligand-field splitting of the $\operatorname{Eu}\left({ }^{7} \mathrm{~F}_{1}\right)$ sublevel and sign of the $\mathrm{B}_{2}^{0}$ parameter. Right:

${ }^{5} \mathrm{D}_{0} \rightarrow{ }^{7} \mathrm{~F}_{1}$ transition in $\left[\mathrm{Eu}_{2}\left(\mathrm{~L}^{\mathrm{C} 2}\right)_{3}\right]$ (redrawn from ref. [21], see Fig. 9 for formula)
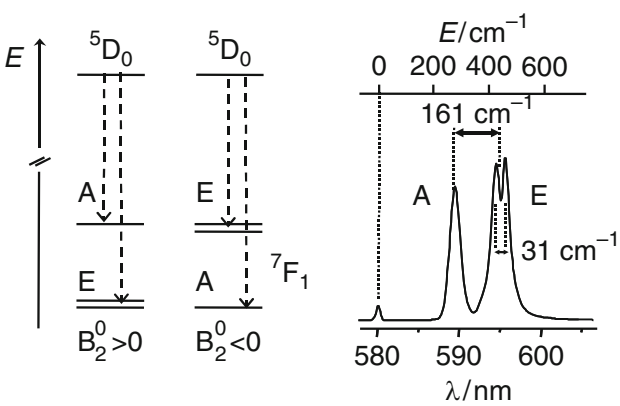
hypersensitive transitions, for instance, $\mathrm{Eu}\left({ }^{5} \mathrm{D}_{0} \rightarrow{ }^{7} \mathrm{~F}_{2}\right)$; in addition, the ${ }^{7} \mathrm{~F}_{2}$ level corresponds to an energy range $\left(1,000-1,500 \mathrm{~cm}^{-1}\right)$ in which the density of phonon states is usually large. On the other hand, these satellites provide useful information on the strength of the Ln-ligand bond: the larger this strength, the more intense the satellites [35].

\subsection{Solvation State of the Metal Ion}

Quenching of the lanthanide luminescence by high-energy vibrations is a major concern in the design of highly luminescent probes. Multi-phonon deactivation is very sensitive to the metal-ligand distance and the phenomenon can be reasonably modeled by Förster's dipole-dipole mechanism [2]. The more phonons needed to bridge the energy gap, the less likely the quenching phenomenon to occur. Table 10 illustrates this phenomenon for solutions of complexes in water $\left(\tilde{v}(\mathrm{O}-\mathrm{H})=3,600 \mathrm{~cm}^{-1}\right)$ and deuterated water $\left(\tilde{v}(\mathrm{O}-\mathrm{D})=2,200 \mathrm{~cm}^{-1}\right)$ by listing the lifetimes of the excited level in these two solvents.

Although detrimental to the emission intensity, vibrational quenching allows one to assess the number of water molecules $q$ interacting in the inner-coordination sphere. Several phenomenological equations have been proposed, based on the assumptions that $\mathrm{O}-\mathrm{D}$ oscillators contribute little to deactivation and that all the other deactivation paths are the same in water and in deuterated water and can henceforth be determined by measuring the lifetime in the deuterated solvent. An important point for their application is to make sure that quenching by solvent vibrations is by far the most important deactivation process in the molecule. If other temperature-dependent phenomena (e.g., phonon-assisted back transfer) are operating, these relationships become unreliable. This has often been observed with $\mathrm{Tb}^{\mathrm{III}}$ [27].

Table 10 Illustration of the energy gap law with respect to quenching of the $\mathrm{Ln}^{\mathrm{III}}$ luminescence by high-energy vibrations. Samples are dilute solutions of perchlorates or triflates at room temperature [11]

\begin{tabular}{llllll}
\hline Ln & $\Delta E / \mathrm{cm}^{-1}$ & No. of phonons & Lifetime $/ \mu \mathrm{s}$ & \\
\hline & & $\mathrm{OH}$ & $\mathrm{OD}$ & $\mathrm{H}_{2} \mathrm{O}$ & $\mathrm{D}_{2} \mathrm{O}$ \\
$\mathrm{Gd}$ & 32,100 & 9 & 15 & 2,300 & n.a. \\
$\mathrm{Tb}$ & 14,800 & 4 & 7 & 467 & 3,800 \\
$\mathrm{Eu}$ & 12,300 & $3-4$ & $5-6$ & 108 & 4,100 \\
$\mathrm{Yb}$ & 10,250 & 3 & 4.5 & $0.17^{\mathrm{a}}$ & 3.95 \\
$\mathrm{Dy}$ & 7,850 & $2-3$ & $3-4$ & 2.6 & 42 \\
$\mathrm{Sm}$ & 7,400 & 2 & 3 & 2.7 & 60 \\
$\mathrm{Er}$ & 6,600 & 2 & 3 & n.a. & 0.37 \\
$\mathrm{Nd}$ & 5,400 & $1-2$ & $2-3$ & 0.031 & 0.14 \\
\hline
\end{tabular}

${ }^{a}$ Estimated from quantum yields in water and deuterated water and from $\tau\left(\mathrm{D}_{2} \mathrm{O}\right)$ 
Altogether, such relationships, which exist for $\mathrm{Nd}^{\mathrm{III}}, \mathrm{Sm}^{\mathrm{III}}, \mathrm{Eu}^{\mathrm{III}}, \mathrm{Tb}^{\mathrm{III}}, \mathrm{Dy}{ }^{\mathrm{III}}$, and $\mathrm{Yb}^{\mathrm{III}}$, are to be used with care and bearing in mind their peculiar calibration. The general form of these relationships is:

$$
\begin{gathered}
q=A \times\left(\Delta k_{\mathrm{obs}}-B\right)-C, \\
\Delta k_{\mathrm{obs}}=k_{\mathrm{H} 2 \mathrm{O}}-k_{\mathrm{D} 2 \mathrm{O}}=1 / \tau\left(\mathrm{H}_{2} \mathrm{O}\right)-1 / \tau\left(\mathrm{D}_{2} \mathrm{O}\right),
\end{gathered}
$$

where $A, B$, and $C$ are phenomenological Ln-depending (and sometimes liganddepending) parameters determined using series of compounds with known hydration numbers. Parameter $A$ describes the inner-sphere contribution to the quenching, parameter $C$ the outer-sphere contribution of closely diffusing solvent molecules, while the corrective factor $B$, which has the same units as $k$, accounts for the presence of other deactivating vibrations (e.g., $\mathrm{N}-\mathrm{H}$ or $\mathrm{C}-\mathrm{H}$ oscillators). Here are the most reliable relationships for $\mathrm{H}_{2} \mathrm{O}$ (and $\mathrm{MeOH}$ ):

$$
\begin{aligned}
& q_{\mathrm{Eu}}\left(\mathrm{H}_{2} \mathrm{O}\right)=1.11 \times\left(\Delta k_{\mathrm{obs}}-0.31\right) \quad \Delta k_{\mathrm{obs}} \text { in } \mathrm{ms}^{-1}, \quad[36] \\
& q_{\mathrm{Eu}}\left(\mathrm{H}_{2} \mathrm{O}\right)=1.2 \times\left(\Delta k_{\mathrm{obs}}-0.25-1.20 q^{\mathrm{NH}}-0.075 q^{\mathrm{CONHR}}\right) \\
& \Delta k_{\mathrm{obs}} \text { in } \mathrm{ms}^{-1},[37]
\end{aligned}
$$

with $q^{\mathrm{NH}}$ being the number of $\mathrm{N}-\mathrm{H}$ oscillators in the first coordination sphere and $q^{\text {CONHR }}$ the number of coordinated amide groups.

$$
\begin{gathered}
q_{\mathrm{Tb}}\left(\mathrm{H}_{2} \mathrm{O}\right)=5.0 \times\left(\Delta k_{\mathrm{obs}}-0.06\right) \quad \Delta k_{\mathrm{obs}} \text { in } \mathrm{ms}^{-1}, \text { [37] } \\
q_{\mathrm{Yb}}\left(\mathrm{H}_{2} \mathrm{O}\right)=1.0 \times\left(\Delta k_{\mathrm{obs}}-0.20\right) \quad \Delta k_{\mathrm{obs}} \text { in } \mu \mathrm{s}^{-1},[37] \\
q_{\mathrm{Yb}}(\mathrm{MeOH})=2.0 \times\left(k_{\mathrm{MeOH}}-k_{\mathrm{CD} 3 \mathrm{OD}}-0.1\right) \quad \Delta k_{\mathrm{obs}} \text { in } \mu \mathrm{s}^{-1} .
\end{gathered}
$$

A flaw to avoid is to use (33)-(37) with $\tau_{\text {obs }}\left(\mathrm{D}_{2} \mathrm{O}\right)$ set equal to the observed lifetime measured on the hydrated sample at $77 \mathrm{~K}$ because it is not granted that all vibrational quenching is switched off at this temperature.

Equations calibrated with polyaminocarboxylates and relying on the sole determination of $\tau\left(\mathrm{H}_{2} \mathrm{O}\right)$ have also been suggested, but they are less reliable:

$$
\begin{array}{cc}
q_{\mathrm{Nd}}\left(\mathrm{H}_{2} \mathrm{O}\right)=0.36 \times k_{\mathrm{obs}}-2.0 & k_{\mathrm{obs}} \text { in } \mu \mathrm{s}^{-1},[38] \\
q_{\mathrm{Sm}}\left(\mathrm{H}_{2} \mathrm{O}\right)=25.4 \times k_{\mathrm{obs}}-0.37 & k_{\mathrm{obs}} \text { in } \mu \mathrm{s}^{-1},[39] \\
q_{\mathrm{Dy}}\left(\mathrm{H}_{2} \mathrm{O}\right)=21.1 \times k_{\mathrm{obs}}-0.6 & k_{\mathrm{obs}} \text { in } \mu \mathrm{s}^{-1} .[39]
\end{array}
$$


Establishing $q$ for $\mathrm{Nd}^{\mathrm{III}}$ is really problematic and other relationships have been put forward, which do not yield very satisfying results, owing to too limited calibration range, as, by the way, for (38)-(40).

The best way to minimize vibration-induced deactivation processes is to design a rigid metal-ion environment, devoid of high-energy vibrations and protecting the $\mathrm{Ln}^{\mathrm{III}}$ ion from solvent interactions. Such an environment also contributes to reduce collision-induced deactivation in solution. Further protection may be gained by inserting the luminescent edifice into micelles, a strategy used in bioanalyses [23]. Recent reports have also demonstrated a considerable weakening of the quenching ability of $\mathrm{O}-\mathrm{H}$ vibrations if the coordinated water molecules are involved in strong intra- or inter-molecular H-bonding. Combining this effect with encapsulation into a rigid receptor turns the weakly emitting aqua ions into entities with sizeable luminescence.

\subsection{Energy Transfers: Donor-Acceptor Distances and Control of the Photophysical Properties of the Acceptor by the Donor}

Distances between a chromophore and a metal-ion site, as well as between metal ions, may be inferred from the determination of energy transfer efficiency within the frame of Förster's dipole-dipole mechanism. In this case, the following simplified equations hold to estimate the efficiency of transfer between the donor D and the acceptor A:

$$
\eta_{\mathrm{et}}=1-\frac{\tau_{\mathrm{obs}}}{\tau_{0}}=\frac{k_{0}}{k_{\mathrm{obs}}}=\frac{1}{1+\left(R_{\mathrm{DA}} / R_{0}\right)^{6}}
$$

in which $\tau_{\mathrm{obs}}$ and $\tau_{0}$ are the lifetimes of the donor in presence and in absence of the acceptor, respectively, $R_{\mathrm{DA}}$ is the distance between the donor and the acceptor, and $R_{0}$ is the critical distance for $50 \%$ transfer, which depends on (1) an orientation factor $\kappa$ having an isotropic limit of $2 / 3,(2)$ the quantum yield $Q_{\mathrm{D}}$ of the donor (in absence of the acceptor), (3) the refractive index $n$ of the medium, and (4) the overlap integral $J$ between the emission spectrum $E(\tilde{v})$ of the donor and the absorption spectrum $\varepsilon(\tilde{v})$ of the acceptor:

$$
\begin{aligned}
R_{0}^{6} & =8.75 \times 10^{-25}\left(\kappa^{2} \cdot Q_{\mathrm{D}} \cdot n^{-4} \cdot J\right), \\
J & =\frac{\int \varepsilon(\tilde{v}) \cdot E(\tilde{v}) \cdot(\tilde{v})^{-4} \mathrm{~d} \tilde{v}}{E(\tilde{v}) \mathrm{d} \tilde{v}} .
\end{aligned}
$$

Estimation of $R_{0}$ is, therefore, accessible from the experimental optical and structural properties of the system. If a crystal structure is at hand, the problem 
simplifies in that $R_{\mathrm{DA}}$ is known and if the lifetimes of (41) can be determined, then calculation of $R_{0}$ is straightforward. Energy transfer between lanthanide probes has allowed determining the distance between $\mathrm{Ca}^{\mathrm{II}}$ and $\mathrm{Zn}^{\mathrm{II}}$ ions in proteins (substituted by $\mathrm{Ln}^{\mathrm{III}}$ ions), as well as the distance between tryptophan chromophores and these metal-ion sites [40].

Another interesting application of directional energy transfer is the control of the photophysical properties of a metal ion (nd or $4 \mathrm{f}$ ) by another one. For instance, $\mathrm{Cr}^{\mathrm{III}}$ can be used to populate the excited state of $\mathrm{Nd}^{\mathrm{III}}$ or $\mathrm{Yb}^{\mathrm{III}}$. If the rate constant of the energy transfer is fast enough and if $k_{\mathrm{obs}}(\mathrm{Cr})<<k_{\mathrm{obs}}(\mathrm{Ln})$, then the excited $\mathrm{Ln}^{\mathrm{III}}$ ions will decay with an apparent lifetime equal to the (long) lifetime of the $3 \mathrm{~d}$ partner. In this case, the lifetime on $\mathrm{Nd}^{\mathrm{III}}$ and $\mathrm{Yb}^{\mathrm{III}}$ can be "shifted" in the millisecond range, which is an advantage for time-resolved detection $[41,42]$.

\subsection{FRET Analysis}

In fact, FRET (Förster resonant energy transfer) analysis has the same basis as the energy transfer described in the above section. It is used either in simple bioanalyses or to detect protein interactions and DNA hybridization. Its principle is shown on Fig. 14 in the case of a homogeneous immunoassay [43].

In homogeneous immunoassays, the analyte is biochemically coupled to two specific antibodies labeled one with a LLB and the other by an organic acceptor. Emission from the organic acceptor is detected in time-resolved mode because the population of its excited state by intramolecular transfer from the LLB shifts its lifetime in the millisecond range. In this way, it is easy to discriminate between the luminescence emitted by uncoupled and coupled antibody molecules labeled with A; similarly, since the luminescence of A is spectrally different from that of the LLB, interference from $\mathrm{Ln}^{\mathrm{III}}$ luminescence emitted by the uncoupled antibody labeled with the $\operatorname{Ln}^{\mathrm{III}}$ chelate is also discriminated. There is, therefore, no need to wash out unused reactants. A method using FRET for the

Fig. 14 Principle of a homogeneous immunoassay based of FRET technology (redrawn from Ref. [43])

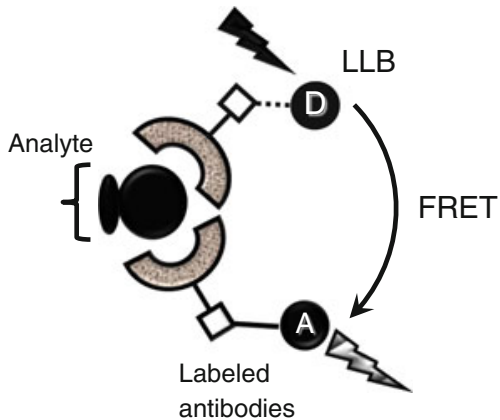


determination of quantum yields of lanthanide chelates and organic dyes has also been described [44].

\subsection{Ligand Exchange Kinetics}

As a starting point, assume that two $\mathrm{Ln}^{\mathrm{III}}$ complexes are present simultaneously in solution at equilibrium and are related by a ligand exchange process. The timedependence of the luminescence emission following an excitation pulse will depend on the rate of chemical exchange relative to the photophysical deactivation rates. If the exchange is slow or fast, no information can be gathered from lifetime measurements. However, if the chemical exchange process occurs at a rate comparable to the de-excitation rate, the time dependence of the luminescence decay is a function of both the excited state lifetimes and the interconversion rate. Equations have been worked out and the exchange kinetics of several $\mathrm{Eu}^{\mathrm{III}}$ and $\mathrm{Tb}^{\mathrm{III}}$ complexes has been elucidated. The $\mathrm{Eu}^{\mathrm{III}}$ ion lends itself more easily to such experiments because selective excitation of one species through the ${ }^{5} \mathrm{D}_{0} \leftarrow{ }^{7} \mathrm{~F}_{0}$ transition can be easily achieved [45, 46].

\subsection{Analytical Probes}

In analytical applications, the $\mathrm{Ln}^{\mathrm{III}}$ absorption or emission properties are either simply detected or modulated by a process depending on the concentration of the analyte, itself reversibly binding to the lanthanide tag. If absorption is used, hypersensitive transitions (Table 4) are good reporters in view of their sensitivity to minute changes in the $\mathrm{Ln}^{\mathrm{III}}$ environment. When it comes to luminescence, a much more sensitive technique (especially if time-resolved detection is used), there are several ways of modulating the emission (Fig. 15). One obvious way is to modulate the solvation in the first coordination sphere (a); alternatively, interaction of the analyte with the ligand molecules may modify the energy transfer ability of the latter (b), and the analyte itself may transfer energy onto the reporter ion (c). Note that situations may be reverse, in that either sensitization or quenching may be induced. Cations, anions, $\mathrm{pH}, \mathrm{pO}_{2}$, aromatic molecule sensors have been designed along these lines, while time-resolved immunoassays often take advantage of FRET technology [23].

Molecular interaction between the luminescent tag and other molecules present in solution can also result in luminescence quenching and quantitative investigation of the phenomenon provides both analytical and photophysical information. In collisional (dynamic) quenching, the quencher molecule diffuses to the luminescent probe during the lifetime of the excited state; upon collision, the latter returns to the ground state without emission of light. The average distance that a molecule having 
Fig. 15 Modulation of lanthanide luminescence by an analyte through reversible binding: (a) removal of solvent quenching, (b) modulation of the ligand ability to transfer energy onto the $\mathrm{Ln}^{\mathrm{III}}$ ion, and (c) binding of a sensitizing analyte to the ligand(s). Redrawn from [42]
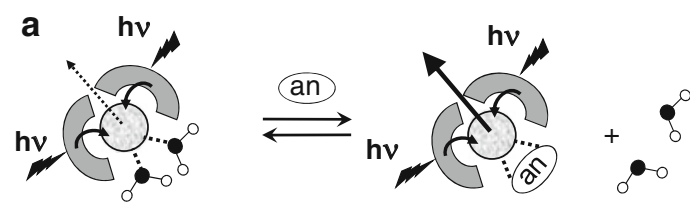

b
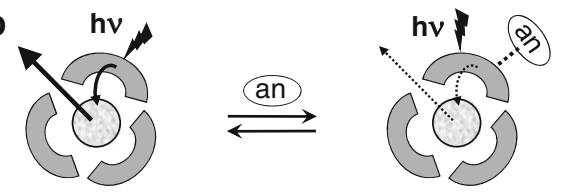

C

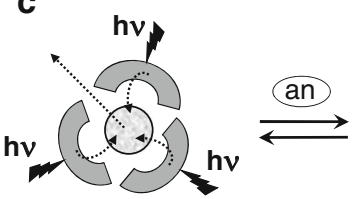

a diffusion coefficient $D$ can travel in solution during the lifetime of the excited state is given by:

$$
\bar{x}=\sqrt{2 D \tau_{\mathrm{obs}}}
$$

A typical collisional quencher is molecular oxygen which has a diffusion coefficient of $2.5 \times 10^{-5} \mathrm{~cm}^{2} \cdot \mathrm{s}^{-1}$ in water at $298 \mathrm{~K}$. During the lifetime of the $\mathrm{Eu}\left({ }^{5} \mathrm{D}_{0}\right)$ level, typically $1 \mathrm{~ms}$, it can, therefore, diffuse over $2.2 \mu \mathrm{m}$, that is a distance comparable to the size of a biological cell. In some instances, a nonluminescent complex may result from the collision (static quenching), similar to the cases shown on Fig. 15. Stern and Volmer have worked out the equation for dynamic quenching:

$$
\frac{E_{0}}{E}=1+K_{\mathrm{D}}[Q]=1+k_{q} \tau_{0}[Q]
$$

in which $K_{\mathrm{D}}$ is the dynamic quenching constant, $k_{\mathrm{q}}$ the bimolecular rate constant, $E_{0}$ and $E$ the emission intensities in absence and in presence of quencher, respectively, and $\tau_{0}$ the observed lifetime in absence of quencher. When collisional quenching occurs, the lifetime decreases in parallel to the luminescence intensity:

$$
\frac{E_{0}}{E}=\frac{\tau_{0}}{\tau} .
$$



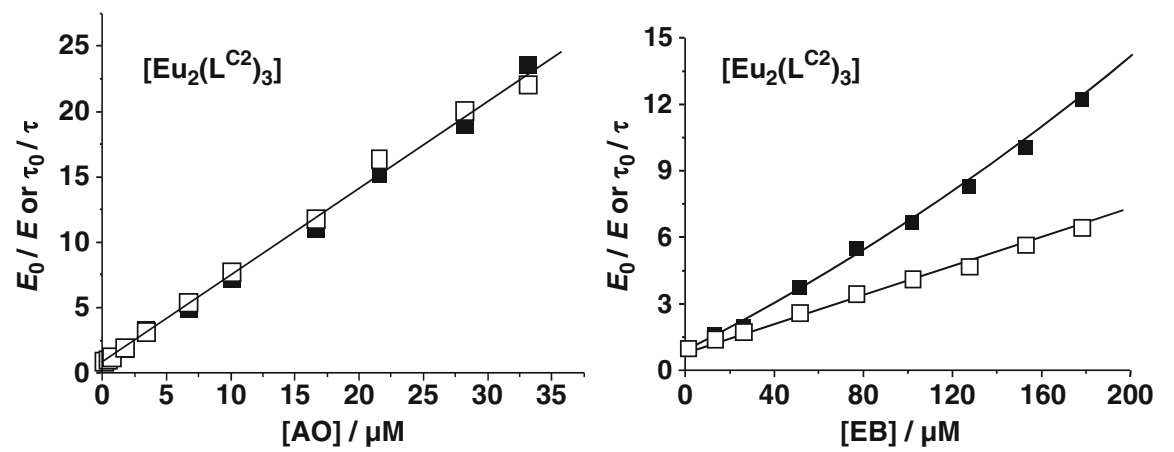

Fig. 16 Stern-Volmer plots for the quenching of the luminescence of $\left[\mathrm{Eu}_{2}\left(\mathrm{~L}^{\mathrm{C} 2}\right)_{3}\right]$ by acridine orange (left) and ethidium bromide (right) in Tris- $\mathrm{HCl}$ buffer ( $\mathrm{pH}$ 7.4). Open squares: lifetimes, solid squares: intensities. Redrawn after [47]

A similar equation can be derived for static quenching, with $K_{\mathrm{S}}$ being the static quenching constant:

$$
\frac{E_{0}}{E}=1+K_{\mathrm{S}}[Q]
$$

When both dynamic and static quenching occurs, the equations combine into:

$$
\begin{gathered}
\frac{E_{0}}{E}=\left(1+K_{\mathrm{D}}[Q]\right)\left(1+K_{\mathrm{S}}[Q]\right), \\
\frac{E_{0}}{E}-1=\left(K_{\mathrm{D}}+K_{\mathrm{S}}\right)[Q]+K_{\mathrm{D}} K_{\mathrm{S}}[Q]^{2} .
\end{gathered}
$$

Here again, (45) holds and luminescence intensities may be substituted with lifetimes. It turns out from these equations that if the Stern-Volmer plot is linear, it reflects the sole presence of dynamic quenching. This is, for instance, the case for the quenching of the bimetallic $\left[\mathrm{Eu}_{2}\left(\mathrm{~L}^{\mathrm{C} 2}\right)_{3}\right]$ helicate with acridine orange $(\mathrm{AO})$, as shown on Fig. 16. The corresponding constants are $K_{D}=6.7(1) \times 10^{5} \mathrm{M}^{-1}$ and $k_{q}=2.7(1) \times 10^{8} \mathrm{M}^{-1} \mathrm{~s}^{-1}$. On the other hand, quenching of the same chelate with ethidium bromide (EB) is typical of both dynamic and static quenching with $K_{D}=3.0(1) \times 10^{4} \mathrm{M}^{-1}, K_{S}=2.0(1) \times 10^{3} \mathrm{M}^{-1}$ and $k_{q}=1.23(4) \times 10^{7} \mathrm{M}^{-1}$ $\mathrm{s}^{-1}$. The bimolecular rate constants are relatively small compared to diffusion $(\approx$ $10^{10} \mathrm{M}^{-1} \mathrm{~s}^{-1}$ ) because of the shielding of the $\mathrm{Eu}{ }^{\mathrm{III}}$ ion embedded inside the helical edifice. This quenching has been taken advantage of to develop a versatile and robust method for the detection of various types of DNA and of PCR products [47].

Acknowledgments This work is supported through grants from the Swiss National Science Foundation. The authors are grateful to Frédéric Gumy for his help in recording luminescence data and to Dr Jonathan Dumke for pertinent comments. 


\section{Appendices}

\subsection{Site Symmetry Determination from $E u^{I I I}$ Luminescence Spectra}

The exact site symmetry can be determined usually only if polarization measurements are made, that is, on single crystals. A light with $\sigma$ polarization has its electric vector perpendicular to the crystallographic $c$ axis and its magnetic vector parallel to it; the reverse holds for $\pi$ polarization. The following scheme sketches how the site symmetries other than cubic $\left(T_{x}, O_{x}\right.$, see Table 3$)$ may be found from $\mathrm{Eu}\left({ }^{5} \mathrm{D}_{0}\right)$ emission spectra. This is the simplest procedure since ${ }^{5} \mathrm{D}_{0}$ is non-degenerate. However, site symmetry can be worked out with other luminescent ions as well and, also, from absorption spectra.

\section{Number of components in the transition}

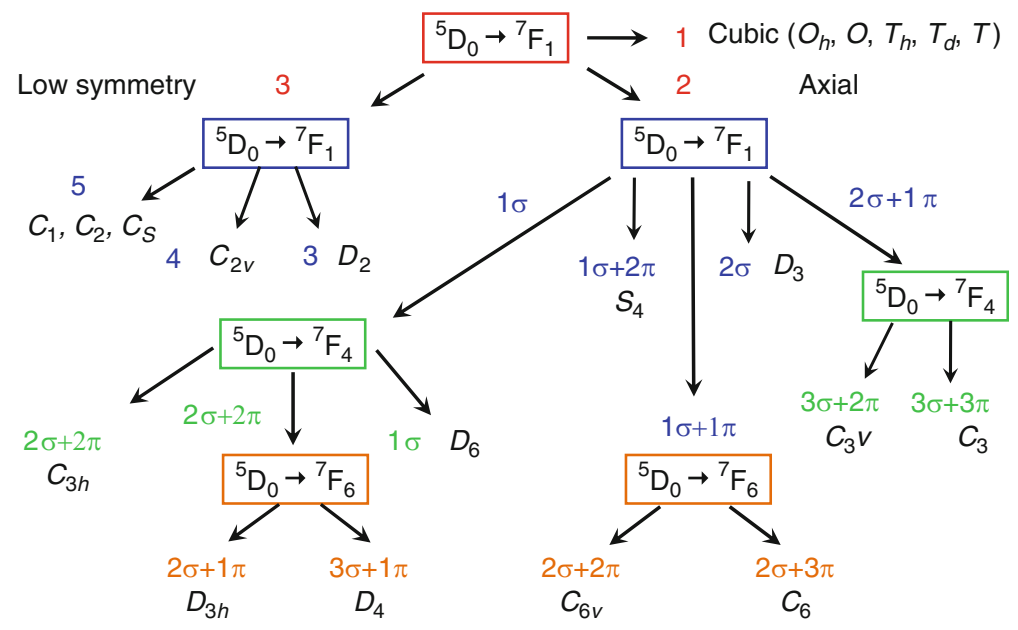

\subsection{Examples of Judd-Ofelt Parameters}

Table 11 Judd-Ofelt parameters for $\mathrm{Ln}^{\mathrm{III}}$ aqua ions in dilute acidic solution [8]

\begin{tabular}{llll}
\hline $\mathrm{Ln}$ & $10^{20} \Omega_{2} / \mathrm{cm}^{2}$ & $10^{20} \Omega_{4} / \mathrm{cm}^{2}$ & $10^{20} \Omega_{6} / \mathrm{cm}^{2}$ \\
\hline $\mathrm{Pr}$ & 32.6 & 5.7 & 32.0 \\
$\mathrm{Nd}$ & 0.93 & 5.00 & 7.91 \\
$\mathrm{Sm}$ & 0.91 & 4.13 & 2.70 \\
$\mathrm{Eu}$ & 1.46 & 6.66 & 5.40 \\
$\mathrm{Gd}$ & 2.56 & 4.70 & 4.73 \\
$\mathrm{~Tb}$ & 0.004 & 7.19 & 3.45 \\
$\mathrm{Dy}$ & 1.50 & 3.44 & 3.46 \\
$\mathrm{Ho}$ & 0.36 & 3.14 & 3.07 \\
$\mathrm{Er}$ & 1.59 & 1.95 & 1.90 \\
$\mathrm{Tm}$ & 0.80 & 2.08 & 1.86 \\
\hline
\end{tabular}


Table 12 Judd-Ofelt parameters for $\mathrm{Nd}^{\mathrm{III}}$ in selected environments [11, 48]

\begin{tabular}{llll}
\hline Sample & $10^{20} \Omega_{2} / \mathrm{cm}^{2}$ & $10^{20} \Omega_{4} / \mathrm{cm}^{2}$ & $10^{20} \Omega_{6} / \mathrm{cm}^{2}$ \\
\hline $\mathrm{Nd}: \mathrm{YAG}$ & 0.37 & 2.29 & 5.97 \\
$\mathrm{Cs} 3\left[\mathrm{Nd}(\mathrm{dpa})_{3}\right] / \mathrm{H}_{2} \mathrm{O}$ & 7.13 & 3.78 & 13.21 \\
$\mathrm{Nd}(\mathrm{ClO})_{4} / \mathrm{MeCN}$ & 1.2 & 7.7 & 7.8 \\
$\mathrm{Nd}\left(\mathrm{ClO}_{4}\right)_{3} / \mathrm{DMF}$ & 1.2 & 8.9 & 8.4 \\
$\mathrm{Nd}\left(\mathrm{NO}_{3}\right)_{3} / \mathrm{MeCN}$ & 11.8 & 2.1 & 6.6 \\
$\mathrm{Nd}\left(\mathrm{NO}_{3}\right)_{3} / \mathrm{DMF}$ & 6.7 & 5.0 & 7.6 \\
\hline
\end{tabular}

\subsection{Examples of Reduced Matrix Elements}

Table 13 Doubly reduced matrix elements used in the calculations of the dipole strengths for absorption and emission of $\mathrm{Cs}_{3}\left[\mathrm{Eu}(\mathrm{dpa})_{3}\right]$, from [7]

\begin{tabular}{llllll}
\hline Transition & Element & Value & Transition & Element & Value \\
\hline${ }^{5} \mathrm{D}_{2} \leftarrow{ }^{7} \mathrm{~F}_{0}$ & $\left|\left\langle\Psi\left\|U^{2}\right\| \Psi^{\prime}\right\rangle\right|^{2}$ & 0.0008 & ${ }^{5} \mathrm{D}_{0} \rightarrow{ }^{7} \mathrm{~F}_{2}$ & $\left|\left\langle\Psi\left\|U^{2}\right\| \Psi^{\prime}\right\rangle\right|^{2}$ & 0.0032 \\
${ }^{5} \mathrm{~L}_{6} \leftarrow{ }^{7} \mathrm{~F}_{1}$ & $\left|\left\langle\Psi\left\|U^{6}\right\| \Psi^{\prime}\right\rangle\right|^{2}$ & 0.0090 & ${ }^{5} \mathrm{D}_{0} \rightarrow{ }^{7} \mathrm{~F}_{4}$ & $\left|\left\langle\Psi\left\|U^{4}\right\| \Psi^{\prime}\right\rangle\right|^{2}$ & 0.0023 \\
${ }^{5} \mathrm{~L}_{6} \leftarrow{ }^{7} \mathrm{~F}_{0}$ & $\left|\left\langle\Psi\left\|U^{6}\right\| \Psi^{\prime}\right\rangle\right|^{2}$ & 0.0155 & ${ }^{5} \mathrm{D}_{0} \rightarrow{ }^{7} \mathrm{~F}_{6}$ & $\left|\left\langle\Psi\left\|\left.\left|U^{6} \| \Psi^{\prime}\right\rangle\right|^{2}\right.\right.\right.$ & 0.0002 \\
${ }^{5} \mathrm{D}_{4} \leftarrow{ }^{7} \mathrm{~F}_{0}$ & $\left|\left\langle\Psi\left\|U^{4}\right\| \Psi^{\prime}\right\rangle\right|^{2}$ & 0.0011 & & & \\
\hline
\end{tabular}

\subsection{Emission Spectra}

In the following we give typical examples of luminescence spectra of the $\mathrm{Ln}^{\mathrm{III}}$ ions, with emission from the main luminescent levels. Depending on the chemical environment of the ion, the shape of the spectra may differ substantially (for instance, the relative intensity and CF splitting of the bands), but the energy of the transitions remains relatively insensitive; in addition vibronic transitions as well
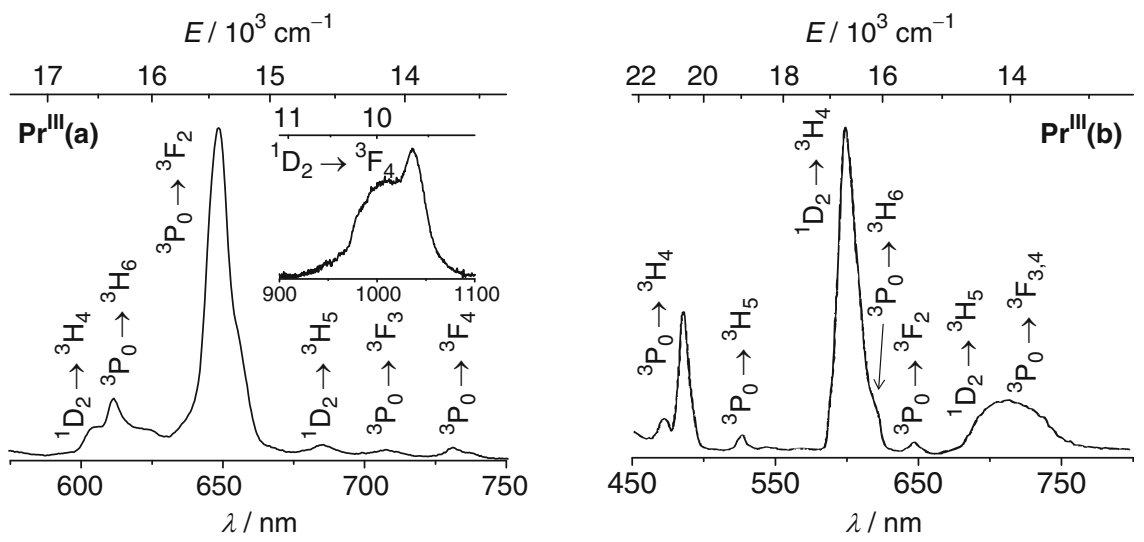

Fig. 17 (continued) 

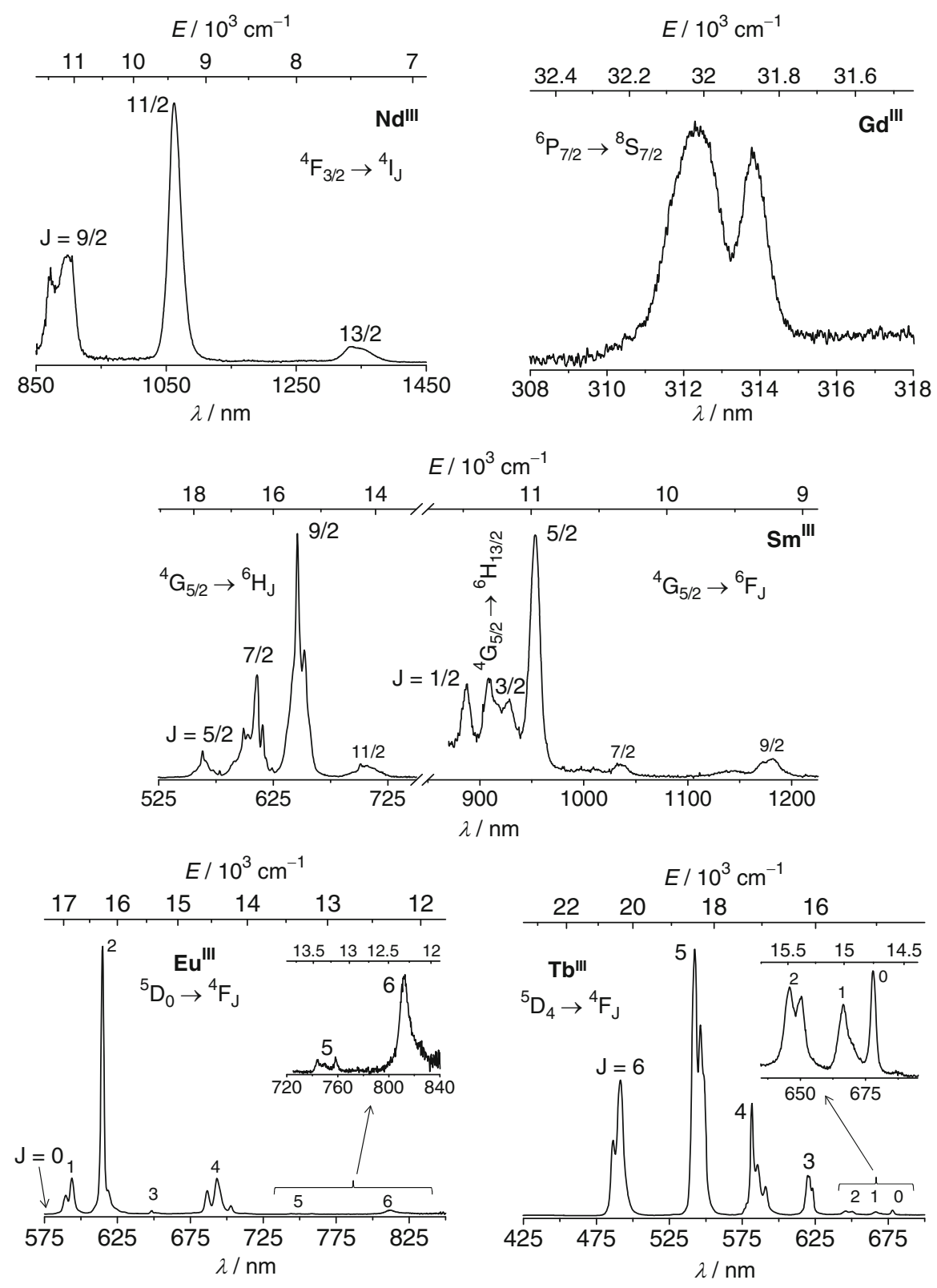

Fig. 17 (continued) 

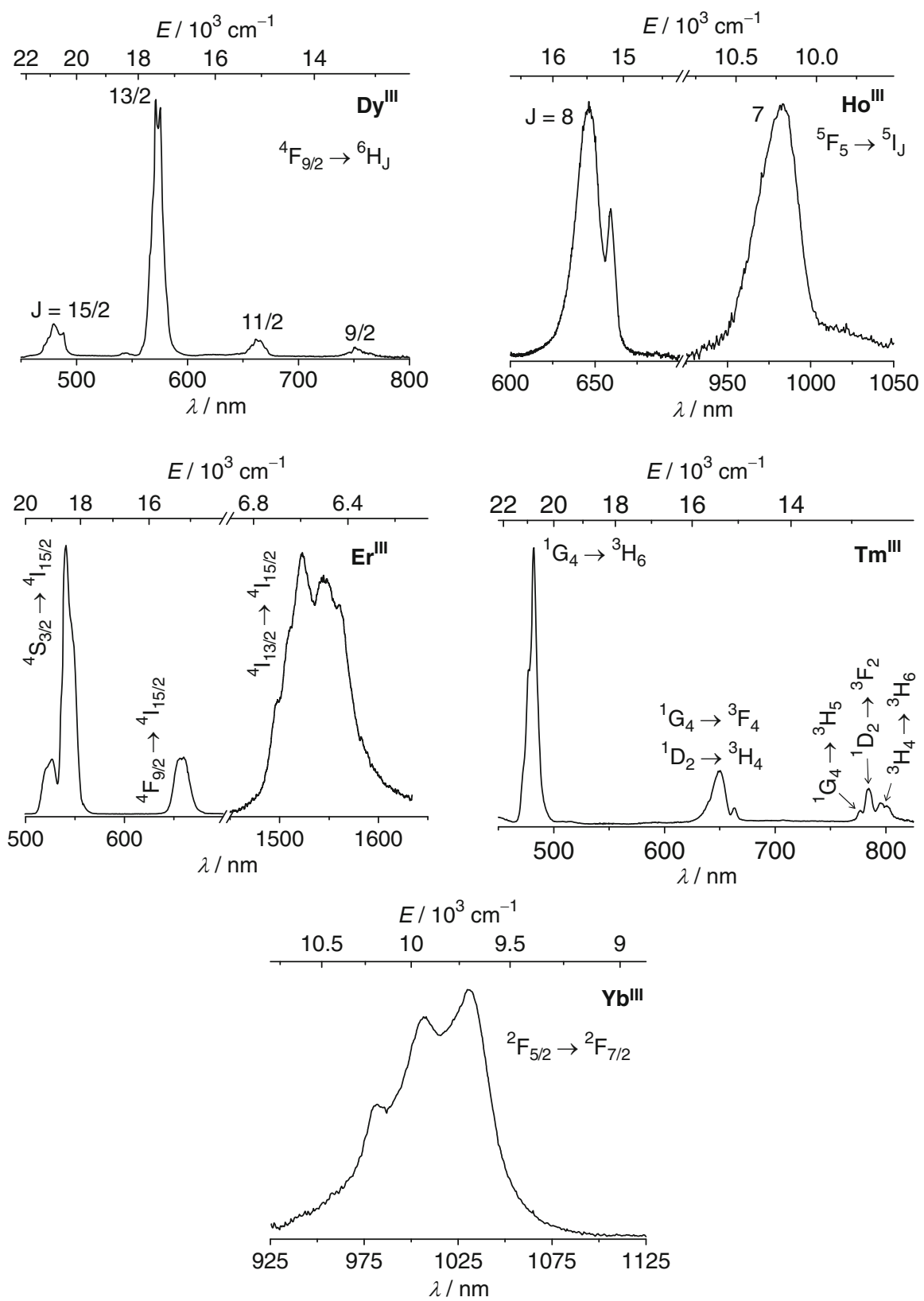

Fig. 17 (continued) Typical examples of $\mathrm{Ln}^{\mathrm{III}}$ emission spectra under ligand excitation (320$340 \mathrm{~nm})$ : microcrystalline samples of dimeric $\left[\mathrm{Ln}(\mathrm{hfa})_{3}(\mathrm{~L})\right]_{2}(\mathrm{~L}=4$-cyanopyridine $\mathrm{N}$-oxide) for $\mathrm{Ln}=\operatorname{Pr}(\mathrm{a}), \mathrm{Nd}, \mathrm{Sm}$, Dy, Ho, Tm, Yb [49]; solutions of $\left[\operatorname{Pr}(\mathrm{b})\left(\mathrm{L}^{1}\right)_{2}\left(\mathrm{NO}_{3}\right)\right]$ in $\mathrm{CH}_{2} \mathrm{Cl}_{2}\left(\mathrm{~L}^{1}=\right.$ dihydrobis-[3-(2-pyridyl)pyrazolyl]borate) [50] and $\mathrm{Cs}_{3}\left[\mathrm{Ln}(\mathrm{dpa})_{3}\right] \approx 2-3.7 \times 10^{-2} \mathrm{M}$ in Tris-HCl $0.1 \mathrm{M}$ (pH 7.4), $\mathrm{Ln}=\mathrm{Eu}, \mathrm{Tb}$ [7]. Emission of $\mathrm{Gd}$ is measured on a microcrystalline sample of $\mathrm{Gd}_{2} \mathrm{O}_{3}$ and of $\mathrm{Er}$ (up-conversion, $\lambda_{\text {exc }}=980 \mathrm{~nm}$ ) on a doped sample of $\mathrm{NaYF}_{4}: \mathrm{Yb} / \mathrm{Er}(18 / 4 \%)$ (this work). All spectra are recorded at room temperature (except for $\mathrm{Tm}, \mathrm{Ho}$, and $\operatorname{Pr}(\mathrm{a})$ in the visible range, $77 \mathrm{~K}$ ), corrected for the instrumental function, and normalized 
as emission from other levels may also show up. In the case of $\operatorname{Pr}^{\mathrm{III}}$, the two spectra shown are quite different because different emissive states are sensitized.

\section{References}

1. Liu G, Jacquier B (2005) Spectroscopic properties of rare earths in optical materials. Tsinghua University Press \& Springer, Beijing \& Heidelberg

2. Hüfner S (1978) Optical spectra of transparent rare earth compounds. Academic, New York

3. Carnall WT, Goodman GL, Rajnak K, Rana RS (1989) A systematic analysis of the spectra of lanthanides doped into single crystal $\mathrm{LaF}_{3}$. J Chem Phys 90:3443-3457

4. Peijzel PS, Meijerink A, Wegh RT, Reid MF, Burdick GW (2005) A complete $4 \mathrm{f}^{n}$ energy level diagram for all trivalent lanthanide ions. J Solid State Chem 178(2):448-453

5. Görller-Walrand C, Binnemans K (1998) Spectral intensities of f-f transitions. In: Gschneidner KA Jr, Eyring L (eds) Handbook on the physics and chemistry of rare earths, vol 25. Elsevier BV, Amsterdam, Ch. 167

6. Walsh BM (2006) Judd-Ofelt theory: principles and practices. In: Di Bartolo B, Forte O (eds) Advances in spectroscopy for lasers and sensing. Springer Verlag, Berlin, pp 403-433

7. Aebischer A, Gumy F, Bünzli JCG (2009) Intrinsic quantum yields and radiative lifetimes of lanthanide tris(dipicolinates). Chem Phys Phys Chem 11:1346-1353

8. Carnall WT (1979) The absorption and fluorescence spectra of rare earth ions in solution. In: Gschneidner KA Jr, Eyring L (eds) Handbook on the physics and chemistry of rare earths, vol 3. Elsevier BV, Amsterdam, Ch. 24

9. Dorenbos P (2000) The f-d transitions of the trivalent lanthanides in halogenides and chalcogenides. J Lumin 91:91-106

10. Shionoya S, Yen WM (1999) Principal phosphor materials and their optical properties. In: Shionoya S, Yen WM (eds) Phosphor handbook. CRC, Boca Raton, Ch. 235

11. Comby S, Bünzli JCG (2007) Lanthanide near-infrared luminescence in molecular probes and devices. In: Gschneidner KA Jr, Bünzli JCG, Pecharsky V (eds) Handbook on the physics and chemistry of rare earths, vol 37. Elsevier BV, Amsterdam, Ch. 24

12. Bredol M, Kynast U, Ronda C (1991) Designing luminescent materials. Adv Mater 3: 361-367

13. Brunet E, Juanes O, Sedano R, Rodriguez-Ubis JC (2002) Lanthanide complexes of polycarboxylate-bearing dipyrazolylpyridine ligands with near-unity luminescence quantum yields: the effect of pyridine substitution. Photochem Photobiol Sci 1:613-618

14. Kleinerman M, Choi SI (1968) Exciton-migration processes in crystalline lanthanide chelates I. Triplet exciton migration in lanthanide chelates of 1, 10-phenantholine. J Chem Phys 49:3901-3908

15. Bünzli JCG, Petoud S, Moret E (1999) Luminescent properties of the Hekis(nitrito)europate (III) ion. Spectrosc Lett 32:155-163

16. Charbonnière LJ, Balsiger C, Schenk KJ, Bünzli JCG (1998) Complexes of p-tert-Butylcalix [5]arene with lanthanides: synthesis, structure and photophysical properties. J Chem Soc Dalton Trans 505-510

17. Werts MHV, Jukes RTF, Verhoeven JW (2002) The emission spectrum and the radiative lifetime of $\mathrm{Eu}^{3+}$ in luminescent lanthanide complexes. Phys Chem Chem Phys 4:1542-1548

18. Piguet C, Bünzli JCG (2010) Self-assembled lanthanide helicates: from basic thermodynamics to applications. In: Gschneidner KA Jr, Bünzli JCG, Pecharsky V (eds) Handbook on the physics and chemistry of rare earths, vol 40. Elsevier BV, Amsterdam, Ch. 247

19. Kleinerman M (1969) Energy migration in lanthanide chelates. J Chem Phys 51:2370-2381

20. de Sà GF, Malta OL, Donega CD, Simas AM, Longo RL, Santa-Cruz PA, da Silva EF (2000) Spectroscopic properties and design of highly luminescent lanthanide coordination complexes. Coord Chem Rev 196:165-195 
21. Bünzli JCG, Chauvin AS, Vandevyver CDB, Song B, Comby S (2008) Lanthanide bimetallic helicates for in vitro imaging and sensing. Ann NY Acad Sci 1130:97-105

22. Pandya S, Yu JH, Parker D (2006) Engineering emissive lanthanide complexes for molecular imaging and sensing. Dalton Trans 2757-2766

23. Hemmilä I, Mukkala VM (2001) Time-resolution in fluorometry technologies, labels, and applications in bioanalytical assays. Crit Rev Clin Lab Sci 38:441-519

24. Latva M, Takalo H, Mukkala VM, Matachescu C, Rodriguez-Ibis JC, Kankare J (1997) Correlation between the lowest triplet state energy level of the ligand and lanthanide(III) luminescence quantum yield. J Lumin 75:149-169

25. Archer RD, Chen HY, Thompson LC (1998) Synthesis, characterization, and luminescence of europium(III) Schiff base complexes. Inorg Chem 37:2089-2095

26. Sato S, Wada M (1970) Relations between intramolecular energy transfer efficiencies and triplet state energies in rare earth $\beta$-diketonates. Bull Chem Soc Jpn 43:1955-1962

27. Deiters E, Song B, Chauvin AS, Vandevyver CDB, Bünzli JCG (2009) Luminescent bimetallic lanthanide bioprobes for cellular imaging with excitation into the visible. Chem Eur J 15:885-900

28. Eaton DF (1988) Reference materials for fluorescence measurements. J Pure Appl Chem 60:1107-1114

29. Melhuish WH (1961) Quantum efficiencies of fluorescence of organic substances: effect of solvent and concentration of the fluorescent solute. J Phys Chem 65:229-235

30. De Mello JC, Wittmann HF, Friend RH (1997) An improved experimental determination of external photoluminescence quantum efficiency. Adv Mater 9:230-232

31. Bünzli JCG, Choppin GR (eds) (1989) Lanthanide probes in life, chemical, and earth sciences: theory and practice. Elsevier, Amsterdam

32. Frey ST, Horrocks WdeW Jr (1995) On correlating the frequency of the ${ }^{5} \mathrm{D}_{0} \leftarrow{ }^{7} \mathrm{~F}_{0}$ transition in $\mathrm{Eu}^{3+}$ complexes with the sum of nephelauxetic parameters for all of the coordinating atoms. Inorg Chim Acta 229:383-390

33. Bünzli JCG, Pradervand GO (1986) The Eu(III) ion as luminescent probe: laser-spectroscopic investigation of the metal ion sites in an 18-crown-6 complex. J Phys Chem Phys 85: 2489-2497

34. Görller-Walrand C, Binnemans K (1996) Rationalization of crystal field parameters. In: Gschneidner KA Jr, Eyring L (eds) Handbook on the physics and chemistry of rare earths, vol 23. Elsevier BV, Amsterdam, Ch. 155

35. Puntus LN, Chauvin AS, Varbanov S, Bünzli JCG (2007) Lanthanide complexes with a calix [8]arene bearing phosphinoyl pendant arms. Eur J Inorg Chem 2315-2326

36. Supkowski RM, Horrocks WdeW Jr (2002) On the determination of the number of water molecules, $q$, coordinated to europium(III) ions in solution from luminescence decay lifetimes. Inorg Chim Acta 340:44-48

37. Beeby A, Clarkson IM, Dickins RS, Faulkner S, Parker D, Royle L, de Sousa AS, Williams JAG, Woods M (1999) Nonradiative deactivation of the excited states of europium, terbium and ytterbium complexes by proximate energy-matched $\mathrm{OH}, \mathrm{NH}$ and $\mathrm{CH}$ oscillators: an improved luminescence method for establishing solution hydration states. J Chem Soc Perkin Trans 2:493-503

38. Kimura T, Kato Y (1998) Luminescence study on determination of the inner-sphere hydration number of Am(III) and Nd(III). J Alloys Compd 271:867-871

39. Kimura T, Kato Y (1998) Luminescence study on hydration states of lanthanide(III) polyaminocarboxylate complexes in aqueous solution. J Alloys Compd 275-277:806-810

40. Horrocks WdeW Jr, Sudnick DR (1981) Lanthanide ion luminescence probes of the structure of biological macromolecules. Acc Chem Res 14:384-392

41. Eliseeva SV, Bünzli JCG (2010) Lanthanide luminescence for functional materials and biosciences. Chem Soc Rev 39:189-227

42. Bünzli JCG, Piguet C (2005) Taking advantage of luminescent lanthanide ions. Chem Soc Rev 34:1048-1077 
43. Bünzli JCG (2009) Lanthanide luminescent bioprobes (LLBs). Chem Lett 38:104-109

44. Xiao M, Selvin PR (2001) Quantum yields of luminescent lanthanide chelates and far-red dyes measured by resonance energy transfer. J Am Chem Soc 123:7067-7073

45. Horrocks WdeW Jr, Arkle VK, Liotta FJ, Sudnick DR (1983) Kinetic parameters for a system at equilibirium from the time course of luminescence emission: a new probe of equilibrium dynamics. Excited-state europium(III) as a species label. J Am Chem Soc 105:3455-3459

46. Ermolaev VL, Gruzdev VP (1984) Novel spectral-kinetic method for investigation of ligand exchange in labile metal complexes in solutions. Inorg Chim Acta 95:179-185

47. Song B, Vandevyver CDB, Deiters E, Chauvin AS, Hemmilä I, Bünzli JCG (2008) A versatile method for quantification of DNA and PCR products based on time-resolved Eu ${ }^{\mathrm{III}}$ luminescence. Analyst 133:1749-1756

48. Bünzli JCG, Vuckovic MM (1984) Solvation of neodymium(iii) perchlorate and nitrate in organic solvents as determined by spectroscopic measurements. Inorg Chim Acta 95:105-112

49. Eliseeva SV, Ryazanov M, Gumy F, Troyanov SI, Lepnev LS, Bünzli JCG, Kuzmina NP (2006) Dimeric complexes of lanthanide(III) hexafluoroacetylacetonates with 4-cyanopyridine $\mathrm{N}$-oxide: synthesis, crystal structure, magnetic, and photoluminescent properties. Eur J Inorg Chem 4809-4820

50. Davies GM, Pope SJA, Adams H, Faulkner S, Ward MD (2005) Photophysical properties of $\operatorname{Pr}(\mathrm{III})$ and $\operatorname{Er}(\mathrm{III})$ complexes of poly-(pyrazolyl)borates. Photochem Photobiol Sci 4:829-834 